\title{
Connectedness of classes of fields and zero-cycles on projective homogeneous varieties
}

\author{
Vladimir Chernousov and Alexander Merkurjev
}

\begin{abstract}
We study the Chow group of zero-dimensional cycles for projective homogeneous varieties of semisimple algebraic groups. We show that in many cases this group has no torsion.
\end{abstract}

\section{Introduction}

Let $X$ be a proper scheme of finite type over a field $F$. A zero-cycle on $X$ is the formal sum $\sum n_{i}\left[x_{i}\right]$ where $n_{i} \in \mathbb{Z}$ and $x_{i}$ are closed (zero-dimensional) points of the variety $X$. The factor group of the group of zero-cycles modulo rational equivalence is called the Chow group of dimension 0 and is denoted by $\mathrm{CH}_{0}(X)$. The assignment $x \mapsto \operatorname{deg}(x)$ extends to the degree homomorphism

$$
\operatorname{deg}: \mathrm{CH}_{0}(X) \rightarrow \mathbb{Z}
$$

The image of deg coincides with $n(X) \mathbb{Z}$ where $n(X)$ is the greatest common divisor of the degrees $\operatorname{deg}(x)=[F(x): F]$ over all closed points $x \in X$. We denote the kernel of deg by $\overline{\mathrm{CH}}_{0}(X)$.

The main purpose of this paper is to present a characteristic free uniform method of computing the group $\overline{\mathrm{CH}}_{0}(X)$ for projective homogeneous varieties of semisimple algebraic groups. The method is based on the idea of parametrization of fields over which $X$ has a point. We illustrate the method by proving that in many cases the group $\overline{\mathrm{CH}}_{0}(X)$ is trivial and give examples of varieties when this group is not trivial. The main results of the paper can be summarized as follows.

Let $X$ be a scheme over $F$. We denote by $\mathcal{A}(X)$ the class of all field extensions $L / F$ such that $X(L) \neq \emptyset$. We say that two fields $L_{0}, L_{1} \in \mathcal{A}(X)$ of the same degree $n$ over $F$ are simply $X$-equivalent if they are members of a continuous family of fields $L_{t} \in \mathcal{A}(X), t \in \mathbb{A}^{1}$, of degree $n$ over $F$ (for a precise definition see $\S 6$ ). We say that $L$ and $L^{\prime}$ are $X$-equivalent if they can be connected by a chain of fields $L_{0}=L, L_{1}, \ldots, L_{r}=L^{\prime}$ such that $L_{i}$ and $L_{i+1}$ are simply $X$-equivalent for $i=0, \ldots, r-1$. Furthermore we say that the class $\mathcal{A}(X)$ is connected if every two fields in $\mathcal{A}\left(X_{E}\right)$ of degree $n\left(X_{E}\right)$ over $E$ are $X_{E}$-equivalent over any special extension $E / F$ (see Section 6).

Our first result (Theorem 6.4) asserts that if $X$ is an arbitrary proper scheme over $F$ such that the class $\mathcal{A}(X)$ is connected and $\overline{\mathrm{CH}}_{0}\left(X_{L}\right)=0$ for any field $L \in \mathcal{A}(X)$, then $\overline{\mathrm{CH}}_{0}(X)=0$. Note that the condition $\overline{\mathrm{CH}}_{0}\left(X_{L}\right)=0$ always holds for projective homogeneous varieties $X$. Thus the connectedness of the class $\mathcal{A}(X)$ for such $X$ implies $\overline{\mathrm{CH}}_{0}(X)=0$.

We prove the connectedness of $\mathcal{A}(X)$ for various classes of projective homogeneous varieties. These include: Severi-Brauer varieties, certain generalized Severi-Brauer varieties, quadrics, involution varieties, and projective homogeneous varieties related to groups of exceptional types ${ }^{3,6} D_{4}, G_{2}, F_{4},{ }^{1,2} E_{6}, E_{7}$ with trivial Tits algebras. As an application we get that $\overline{\mathrm{CH}}_{0}(X)=0$,

Received 18 February 2006, accepted in final form 11 May 2006. 2000 Mathematics Subject Classification 20G15, 14M15, $14 \mathrm{M} 17$.

Keywords: algebraic groups, projective homogeneous varieties, zero-cycles.

The first author was supported in part by Canada Research Chairs Program and NSERC Canada Grant G121210944. The second author was supported in part by NSF Grant \#0355166.

This journal is (C) Foundation Compositio Mathematica 2006. 


\section{ZERO-CYCLES}

i.e. the Chow group $\mathrm{CH}_{0}(X)$ is infinite cyclic for all the above-mentioned varieties. We borrow from [Kra05] the idea of using symplectic involutions in the case of generalized Severi-Brauer varieties and involution varieties.

Some of our results were known before under certain restrictions on the characteristic of the ground field $F$. Triviality of $\overline{\mathrm{CH}}_{0}(X)$ in the case of the Severi-Brauer variety $X=S B(A)$ was proven by Panin in [Pan84] if char $F$ does not divide ind $(A)$. Quadrics over fields of characteristic not equal to 2 were considered by Swan [Swa89] and Karpenko [Kar91]. The cases of certain generalized SeveriBrauer varieties and involution varieties were treated by Krashen [Kra05] under the assumption char $F=0$. Involution varieties of algebras of index at most 2 were considered in [Mer95b] by the second author under the assumption $\operatorname{char} F \neq 2$. Petrov et al. [PSZ05], independently, have recently shown that $\overline{\mathrm{CH}}_{0}(X)=0$ for projective homogeneous varieties $X$ related to groups of types $G_{2}, F_{4},{ }^{1} E_{6}$ over fields of characteristic 0 with trivial Tits algebras.

The notion of $X$-equivalence used in this paper as the main technical tool is formulated in terms of field extensions and discrete valuations, so that we avoid the symmetric power constructions used in [Kra05] to describe closed points. Flexibility of the notion of $X$-equivalence allows us not to impose any characteristic restrictions on $F$ (except for the trialitarian $D_{4}$ and $E_{6}$, $E_{7}$ where we assume that the characteristic is not equal to 2 or 3 ). Another advantage of our method is its transparency and shortness. Even for those varieties where the result was already known, our proofs are simpler.

Finally, we remark that most likely our results on algebraic groups of exceptional types are close to optimal. It looks hopeless to weaken restrictions on Tits algebras and prove that $\mathrm{CH}_{0}(X)$ is infinite cyclic for larger classes of projective homogeneous varieties of exceptional groups.

At the end of the paper we give two examples of projective homogeneous varieties $X$ with $\overline{\mathrm{CH}}_{0}(X) \neq 0$ related to algebraic groups of types $A_{1}+A_{1}+A_{1}$ and $B_{3}$ with nontrivial Tits algebras. Note that our first result is the minimal possible since $\overline{\mathrm{CH}}_{0}(X)=0$ for all projective homogeneous varieties $X$ of dimension at most 2 (see Proposition 4.4).

\section{Preliminary facts on algebraic groups}

\subsection{Parabolic subgroups}

Let $G$ be a simple simply connected algebraic group over a field $F$. Fix a maximal torus $T \subset G$ over $F$ and a basis $\Delta$ of the root system $\Sigma=\Sigma(G, T)$ of $G$ with respect to $T$. Recall that for each subset $S \subset \Delta$ one can associate the parabolic subgroup $P_{S}$ in $G$, whose semisimple part is generated by the corresponding root subgroups $U_{ \pm \alpha}$ of $G$ for all roots $\alpha \in S$. It is defined over a separable closure $F_{\text {sep }}$ of $F$ and is called the standard parabolic subgroup of type $S$. Every parabolic subgroup $P$ in $G$ over $F_{\text {sep }}$ is conjugate to a unique standard parabolic subgroup $P_{S}$. We say that $P$ is of type $S$.

Let $X_{S}$ be the variety of all parabolic subgroups of $G$ of type $S$. If $S$ is stable with respect to the so-called *-action of the Galois group $\operatorname{Gal}\left(F_{\text {sep }} / F\right)$ (see [Tit66]), the variety $X_{S}$ is defined over $F$. It has a rational point if and only if $G$ contains an $F$-defined parabolic subgroup of type $S$. If $X_{S}(F) \neq \emptyset$, then we say that $X_{S}$ is isotropic.

\subsection{Tits indices}

Recall [Tit66] that given $G$ one can associate a geometric picture called the Tits index of $G$. It consists of the corresponding Dynkin diagram of $G$ with some vertices circled. The set $S_{0}$ of uncircled vertices corresponds to an $F$-subgroup of $G$ called a semisimple anisotropic kernel. This subgroup is the semisimple part of the centralizer $C_{G}\left(T_{1}\right)$ where $T_{1} \subset G$ is a maximal $F$-split subtorus in $G$. All maximal $F$-split subtori are conjugate over $F$. Hence a semisimple anisotropic kernel of $G$ is defined uniquely up to conjugation. 


\section{Chernousov and A. Merkurjev}

All vertices in the Tits index of $G$ are circled if and only if $G$ is quasi-split over $F$, and none of them is circled if and only if $G$ is $F$-anisotropic. The last occurs if and only if $G$ contains no nontrivial $F$-split subtorus.

Let $S$ be stable with respect to the *-action of $\operatorname{Gal}\left(F_{\text {sep }} / F\right)$. Then the variety $X_{S}$ of parabolic subgroups in $G$ of type $S$ is defined over $F$ and it has a rational point if and only if $S$ contains the subset $S_{0}$ of all uncircled vertices. Hence an $F$-defined parabolic subgroup $P \subset G$ of type $S$ is minimal if and only if $S=S_{0}$; in particular all minimal parabolic subgroups in $G$ have the same type and they are conjugate over $F$.

\subsection{Basic types}

We say that $S$ is a basic type for $G$ if there is a form $H$ of $G$ over a field extension $L / F$ such that $H$ is $L$-isotropic and its minimal parabolic subgroups have type $S$. In other words, a type $S$ is basic if in the classification table [Tit66] of Tits indices there is one whose set of all uncircled vertices coincides with $S$.

\section{Strongly inner forms}

One knows that $G$ is a form of a quasi-split simply connected group $G_{0}$, i.e. there exists a cocycle $\xi \in Z^{1}\left(F\right.$, Aut $\left.G_{0}\right)$ such that $G$ is isomorphic over $F$ to the twisted group ${ }^{\xi} G_{0}$. One says that $G$ is of the same inner type as $G_{0}$ or an inner form of $G_{0}$ if the class $[\xi]$ is contained in $\operatorname{Im}\left[H^{1}\left(F, \bar{G}_{0}\right) \rightarrow\right.$ $H^{1}\left(F\right.$, Aut $\left.\left.G_{0}\right)\right]$ where $\bar{G}_{0}=G_{0} / Z$ is the corresponding adjoint group and $Z \subset G_{0}$ is the center of $G_{0}$. Otherwise $G$ is an outer form of $G_{0}$. One says that $G$ is a strongly inner form of $G_{0}$ if $[\xi]$ is contained in $\operatorname{Im}\left[H^{1}\left(F, G_{0}\right) \rightarrow H^{1}\left(F\right.\right.$, Aut $\left.\left.G_{0}\right)\right]$.

Remark 3.1. The notion of strongly inner forms was first introduced by Tits in [Tit90].

For future reference we need some structure facts on strongly inner forms of a quasi-split simple simply connected group $G_{0}$. In what follows $\xi \in Z^{1}\left(F, G_{0}\right)$ and $G={ }^{\xi} G_{0}$ is the corresponding strongly inner form of $G_{0}$.

\subsection{Quasi-splitness criterion}

Lemma 3.2. The group $G$ is quasi-split if and only if $[\xi]=1$.

Proof. Let $G$ be quasi-split. Since $H^{1}\left(F\right.$, Aut $\left.G_{0}\right)$ classifies all forms of $G_{0}$,

$$
[\xi] \in \operatorname{Ker}\left[H^{1}\left(F, G_{0}\right) \rightarrow H^{1}\left(F, \operatorname{Aut} G_{0}\right)\right] .
$$

The group Aut $G_{0}$ is the semi-direct product of the adjoint group $\bar{G}_{0}$ and the automorphism group Aut $D$ of the Dynkin diagram $D$ of $G_{0}$. It follows that Aut $G_{0}(F) \rightarrow \operatorname{Aut} D(F)$ is surjective, hence the mapping $H^{1}\left(F, \bar{G}_{0}\right) \rightarrow H^{1}\left(F\right.$, Aut $\left.G_{0}\right)$ has trivial kernel. This implies that $[\xi]$ is contained in the kernel of $H^{1}\left(F, G_{0}\right) \rightarrow H^{1}\left(F, \bar{G}_{0}\right)$. Consider now an exact sequence $1 \rightarrow Z \rightarrow G_{0} \rightarrow \bar{G}_{0} \rightarrow 1$. It gives rise to an exact sequence

$$
H^{1}(F, Z) \rightarrow H^{1}\left(F, G_{0}\right) \rightarrow H^{1}\left(F, \bar{G}_{0}\right)
$$

It follows that $\xi$ up to equivalence takes values in $Z$. As $G_{0}$ is simply connected and quasi-split, it contains a maximal quasi-split torus $T \subset G_{0}$. Since $Z \subset T$ and $H^{1}(F, T)=1$, by Hilbert's theorem 90, the result follows.

\subsection{Application of Steinberg's theorem}

Let $C_{1} \subset G$ be a maximal $F$-split torus. The centralizer $C_{G}\left(C_{1}\right)$ is a reductive group over $F$ whose central torus $C$ contains $C_{1}$. Note that if $G_{0}$ is split, we have $C=C_{1}$. However in the 


\section{ZERO-CYCLES}

general case $C \neq C_{1}$. The semisimple part $H=\left[C_{G}\left(C_{1}\right), C_{G}\left(C_{1}\right)\right]$ of $C_{G}\left(C_{1}\right)$ is an $F$-anisotropic simply connected group, not necessarily simple. Its Tits index coincides with the subindex of $G$ generated by uncircled vertices. The group $C_{G}\left(C_{1}\right)$ is an almost direct product of $H$ and $C$, i.e. $H \cap C$ is a finite central subgroup of $H$ and $C_{G}\left(C_{1}\right)=H \cdot C$.

Theorem 3.3. Assume that $H^{1}(F, C / H \cap C)=1$. Then $H$ is a strongly inner form of a quasi-split simply connected group $H_{0}$ over $F$.

Proof. Let $C_{2} \subset H$ be a maximal torus over $F$ and let $T=C \cdot C_{2}$. By dimension count, $T$ is a maximal torus in $G$ over $F$. It easily follows from arguments contained in [Ste65, § 10] (see also [PR94, Propositions 6.18 and 6.19, pp. 338-339]) that there is an F-embedding $\phi: T \rightarrow G_{0}$ such that the class $[\xi]$ is contained in $\operatorname{Im}\left[H^{1}(F, T) \rightarrow H^{1}\left(F, G_{0}\right)\right]$. Since $C \cap H=C \cap C_{2}$ and $H^{1}(F, C / C \cap H)=1$, the mapping $H^{1}\left(F, C_{2}\right) \rightarrow H^{1}(F, T)$ is surjective. Thus, up to equivalence, we may assume that $\xi$ takes values in $C_{2}$.

The centralizer $C_{G_{0}}\left(C_{1}\right)$ is a reductive $F$-subgroup in $G_{0}$ and from the construction of $\phi$ (see the proof in [PR94, Proposition 6.18, p. 339]) it follows that $C_{G_{0}}\left(C_{1}\right)$ and $C_{G}\left(C_{1}\right)$ are isomorphic over $F_{\text {sep }}$. Hence their semisimple parts $H_{0}$ and $H$ are isomorphic over $F_{\text {sep }}$. As all maximal $F$-split tori in $G_{0}$ are conjugate, $C_{G_{0}}\left(C_{1}\right)$ (and hence $H_{0}=\left[C_{G_{0}}\left(C_{1}\right), C_{G_{0}}\left(C_{1}\right)\right]$ ) is a quasi-split group. By construction $H_{0}$ contains $C_{2}$, hence $\xi$ can be viewed as an element in $Z^{1}\left(F, H_{0}\right)$ and then clearly we have $H \simeq{ }^{\xi} H_{0}$.

\subsection{Groups isotropic over a quadratic extension}

Assume that $F$ is infinite and perfect. Let $L / F$ be a quadratic extension making $G$ isotropic. We denote the generator of $\operatorname{Gal}(L / F)$ by $\sigma$. If $P \subset G$ is an $L$-defined standard parabolic subgroup of type $S \subset \Delta$, we denote by $P^{-}$the opposite parabolic subgroup (it is generated by root subgroups $U_{-\alpha}, \alpha \in \Delta \backslash S$, and $\left.U_{ \pm \alpha}, \alpha \in S\right)$. The class $\mathcal{P}$ of parabolic subgroups conjugate to $P$ is called self-opposite if it contains $P^{-}$. Clearly, $P \cap P^{-}$is a reductive part of $P$.

LEMma 3.4. If $\mathcal{P}$ is self-opposite, then it contains an $L$-parabolic subgroup $Q \in \mathcal{P}$ such that $Q \cap \sigma(Q)$ is a reductive part of $Q$.

Proof. See [PR94, Lemma 17', p. 383].

\section{Comparison of two homogeneous varieties}

Proposition 4.1. Let $X$ be a scheme over $F$ and $Y$ be a projective homogeneous variety over $F$ such that $Y_{F(x)}$ is isotropic for every $x \in X$. Then the projection $f: X \times Y \rightarrow X$ induces an isomorphism $f_{*}: \mathrm{CH}_{0}(X \times Y) \rightarrow \mathrm{CH}_{0}(X)$.

Proof. Consider the spectral sequence $[\operatorname{Ros} 96, \S 8]$

$$
E_{p, q}^{1}=\coprod_{x \in X_{(p)}} A_{q}\left(Y_{F(x)}, K_{p}\right) \Rightarrow A_{p+q}\left(X \times Y, K_{0}\right)
$$

for the projection $f$, where $A_{*}\left(X \times Y, K_{*}\right)$ are the $K$-homology groups defined in [Ros96]. It gives an exact sequence

$$
E_{1,0}^{1} \stackrel{\partial}{\rightarrow} E_{0,0}^{1} \rightarrow \mathrm{CH}_{0}(X \times Y) \rightarrow 0 .
$$

We shall identify the terms $E_{n, 0}^{1}$. It is proven in [CGM05, Theorem 7.5] that the Chow motive of the isotropic variety $Y_{F(x)}$ is isomorphic over $F(x)$ to a direct sum of the motive $\mathbb{Z}$ and twisted motives of the form $Z(r)$ for some projective homogeneous variety $Z$ and $r>0$. By the dimension consideration, $A_{0}\left(Z(r), K_{n}\right)=A_{-r}\left(Z, K_{n+r}\right)=0$, hence

$$
A_{0}\left(Y_{F(x)}, K_{n}\right) \simeq A_{0}\left(\operatorname{Spec} F(x), K_{n}\right)=K_{n} F(x),
$$




\section{Chernousov and A. Merkurjev}

where the isomorphism is the push-forward map with respect to the structure morphism $Y_{F(x)} \rightarrow$ Spec $F(x)$. Thus

$$
E_{n, 0}^{1}=\coprod_{x \in X_{(n)}} K_{n} F(x)
$$

and therefore $\mathrm{CH}_{0}(X \times Y)=\operatorname{Coker}(\partial) \simeq \mathrm{CH}_{0}(X)$.

Corollary 4.2. Let $X$ and $Y$ be two projective homogeneous varieties over $F$ such that each of them is isotropic over the residue field of every point of another. Then $\mathrm{CH}_{0}(X) \simeq \mathrm{CH}_{0}(Y)$ and $\overline{\mathrm{CH}}_{0}(X) \simeq \overline{\mathrm{CH}}_{0}(Y)$.

Taking $Y=\operatorname{Spec} F$ in Corollary 4.2 we get the following result.

Corollary 4.3. Let $X$ be an isotropic projective homogeneous variety. Then $\overline{\mathrm{CH}}_{0}(X)=0$.

Let $X$ be a variety over a field $F$. Consider the Brown-Gersten-Quillen spectral sequence

$$
E_{2}^{p, q}=A^{p}\left(X, K_{-q}\right) \Rightarrow K_{-p-q}(X),
$$

where $A^{p}\left(X, K_{-q}\right)$ is the $K$-cohomology group and $K_{n}(X)$ is the $K$-group of $X$ (see [Qui73, $\S 7$ ). If $X$ is projective, the group $E_{2}^{0,-1}=A^{0}\left(X, K_{1}\right)$ of regular invertible functions on $X$ is equal to $K_{1}(F)=F^{\times}$. Since the composite $K_{1}(F) \rightarrow K_{1}(X) \stackrel{e}{\rightarrow} A^{0}\left(X, K_{1}\right)$ is the identity, the edge homomorphism $e$ is surjective. In particular, all the differentials going from $E_{*}^{0,-1}$ are trivial. If $d=$ $\operatorname{dim} X \leqslant 2$, all differentials coming to $E_{*}^{d,-d}$ are trivial; in particular, the edge homomorphism $e^{\prime}: \mathrm{CH}_{0}(X) \rightarrow K_{0}(X)$ is injective.

If $X$ is projective, the degree homomorphism for $\mathrm{CH}_{0}(X)$ is the composition

$$
\mathrm{CH}_{0}(X) \stackrel{e^{\prime}}{\rightarrow} K_{0}(X) \stackrel{f_{*}}{\rightarrow} K_{0}(\operatorname{Spec} F)=\mathbb{Z},
$$

where $f_{*}$ is the push-forward homomorphism with respect to the structure morphism $f: X \rightarrow$ $\operatorname{Spec}(F)$. Therefore, $\operatorname{Ker}\left(e^{\prime}\right) \subset \overline{\mathrm{CH}}_{0}(X)$. If $X$ is a projective homogeneous variety, the group $K_{0}(X)$ is torsion free (see $[\mathrm{Pan} 94])$, hence $\overline{\mathrm{CH}}_{0}(X) \subset \operatorname{Ker}\left(e^{\prime}\right)$ since $\overline{\mathrm{CH}}_{0}(X)$ is a torsion group. Thus $\overline{\mathrm{CH}}_{0}(X)=$ $\operatorname{Ker}\left(e^{\prime}\right)$. In particular we have proven the following proposition.

Proposition 4.4. Let $X$ be a projective homogeneous variety of dimension at most 2 . Then $\overline{\mathrm{CH}}_{0}(X)=0$.

\subsection{Reduction to a basic type}

Let $G$ be a simple simply connected group over $F$. We fix a maximal torus $T \subset G$ over $F$, the corresponding root system $\Sigma=\Sigma(G, T)$, a basis $\Delta \subset \Sigma$ and a subset $S \subset \Delta$. Consider the variety $X_{S}$ of parabolic subgroups in $G$ of type $S$. Let $L=F\left(X_{S}\right)$ be the field of rational functions of $X_{S}$. Since $X_{S}(L) \neq \emptyset$, there is an $L$-defined parabolic subgroup $P$ in $G$ of type $S$. It contains a minimal parabolic subgroup $P^{\prime} \subset P$ of $G$ over $L$ whose type $S^{\prime}$ is a subset in $S$.

Proposition 4.5. There are canonical isomorphisms $\mathrm{CH}_{0}\left(X_{S}\right) \simeq \mathrm{CH}_{0}\left(X_{S^{\prime}}\right)$ and $\overline{\mathrm{CH}}_{0}\left(X_{S}\right) \simeq$ $\overline{\mathrm{CH}}_{0}\left(X_{S^{\prime}}\right)$.

Proof. We show that $X_{S}$ and $X_{S^{\prime}}$ satisfy the conditions of Corollary 4.2. As $S^{\prime} \subset S$, there is a natural map $f: X_{S^{\prime}} \rightarrow X_{S}$ over $F$. Therefore $X_{S}$ is isotropic over $F\left(x^{\prime}\right)$ for every $x^{\prime} \in X_{S^{\prime}}$.

Conversely, let $x \in X_{S}$ and set $E=F(x)$. The set $S^{\prime}$ consists of all uncircled vertices of the Tits index of $G$ over $F(X)$. Therefore $S^{\prime}$ contains all uncircled vertices of the Tits index of $G$ over $E(X)$. Since $E(X) / E$ is purely transcendental, and the Tits index does not change under such extensions, the set $S^{\prime}$ contains all uncircled vertices of the Tits index of $G$ over $E$, i.e. $X_{S^{\prime}}(E) \neq \emptyset$. 


\section{ZERO-CYCLES}

Remark 4.6. The previous proposition allows us to consider basic subsets $S \subset \Delta$ only while computing $\mathrm{CH}_{0}\left(X_{S}\right)$. In fact we have proved even more: it is sufficient to compute $\mathrm{CH}_{0}\left(X_{S}\right)$ for those basic subsets $S$ that appear as sets of uncircled vertices of the Tits indices of $G$ over all field extensions $L / F$. Hence we can ignore a basic type $S$ if it corresponds to an anisotropic kernel of a form $H$ of $G$ which is not strongly inner.

\section{Special fields}

Let $p$ be a prime integer. A field $F$ is called $p$-special if the degree of every finite field extension of $F$ is a power of $p$. We say that $F$ is special if $F$ is $p$-special for some $p$. The following properties of special fields follow directly from the definition.

(i) An algebraic extension of a $p$-special field is $p$-special.

(ii) For every field $F$ and every prime $p$ there is an algebraic field extension $F_{p} / F$ such that the field $F_{p}$ is $p$-special and the degree of any finite subextension $L / F$ of $F_{p} / F$ is prime to $p$.

If $X$ is a scheme over a $p$-special field $F$, then $n(X)$ is a power of $p$ and there is a closed point $x \in X$ such that $\operatorname{deg} x=n(X)$.

The following statement reduces the problem of computing $\mathrm{CH}_{0}(X)$ to the case of schemes over $p$-special fields.

Lemma 5.1. Let $X$ be a proper scheme over $F$. Assume that for any $p$-special field extension $L / F$, the group $\overline{\mathrm{CH}}_{0}\left(X_{L}\right)$ is trivial. Then $\overline{\mathrm{CH}}_{0}(X)=0$.

Proof. Let $a \in \overline{\mathrm{CH}}_{0}(X)$ and fix a prime integer $p$. As the image of $a$ in $\overline{\mathrm{CH}}_{0}\left(X_{F_{p}}\right)$ is trivial, $a$ is trivial over some finite subextension $L / F$ in $F_{p} / F$. Applying the push-forward homomorphism with respect to the morphism $X_{L} \rightarrow X$, we get $[L: F] \cdot a=0$. In particular, the order of $a$ is not divisible by $p$. Since this holds for every prime $p$, we have $a=0$.

The following statement enables us to consider only closed points of minimal degree.

Lemma 5.2. Let $X$ be a proper scheme over a $p$-special field $F$. Assume that for any finite field extension $L / F$, every two closed points of $X_{L}$ of the smallest degree $n\left(X_{L}\right)$ are rationally equivalent. Then $\overline{\mathrm{CH}}_{0}(X)=0$.

Proof. Let $x$ be a closed point of $X$ of the smallest degree $p^{a}=n(X)$ and let $y$ be a closed point of $X$ of degree $p^{b}$. We claim that $[y]=p^{b-a}[x]$ in $\mathrm{CH}_{0}(X)$.

We shall prove the claim by induction on $p^{b}=\operatorname{deg}(y)$. We may assume that $p^{b}>p^{a}$. Choose an intermediate field $F \subset L \subset F(y)$ such that $[L: F]=p$ and a point $y^{\prime} \in X_{L}$ over $y$ of degree $p^{b-1}$. Note that $n\left(X_{L}\right)$ divides $n(X)$ and $n(X)$ divides $p \cdot n\left(X_{L}\right)$. Therefore we have the following two cases.

Case 1: $n\left(X_{L}\right)=n(X)=p^{a}$. Then there is a point $x^{\prime} \in X_{L}$ over $x$ of degree $p^{a}$. By the induction hypothesis applied to $X_{L},\left[y^{\prime}\right]=p^{b-1-a}\left[x^{\prime}\right]$ in $\mathrm{CH}_{0}\left(X_{L}\right)$. Taking the push-forward homomorphism with respect to $f: X_{L} \rightarrow X$ we get

$$
[y]=f_{*}\left(\left[y^{\prime}\right]\right)=p^{b-1-a} f_{*}\left(\left[x^{\prime}\right]\right)=p^{b-1-a} \cdot p[x]=p^{b-a}[x] .
$$

Case 2: $n\left(X_{L}\right)=p^{a-1}$. Choose a closed point $z^{\prime} \in X_{L}$ of degree $p^{a-1}$ and denote by $z$ its image in $X$. The degree of $z$ is $p^{a}$; in particular, $[z]=[x]$ in $\mathrm{CH}_{0}(X)$. By the induction hypothesis applied to $X_{L},\left[y^{\prime}\right]=p^{(b-1)-(a-1)}\left[z^{\prime}\right]$ in $\mathrm{CH}_{0}\left(X_{L}\right)$. Therefore

$$
[y]=f_{*}\left(\left[y^{\prime}\right]\right)=p^{(b-1)-(a-1)} f_{*}\left(\left[z^{\prime}\right]\right)=p^{b-a}[z]=p^{b-a}[x] .
$$




\section{Chernousov and A. Merkurjev}

The claim is proven. It follows that every zero-cycle $\alpha$ on $X$ is equivalent to $m[x]$ for some $m \in \mathbb{Z}$. If $\operatorname{deg} \alpha=0$, then $m=0$, i.e. $\alpha=0$ in $\mathrm{CH}_{0}(X)$.

\section{Equivalences of field extensions}

Let $F$ be a field and let $\mathcal{A}$ be the class of all field extensions of $F$. For any subclass $\mathcal{B} \subset \mathcal{A}$ and every field $L \in \mathcal{A}$ we write $\mathcal{B}_{L}$ for the subclass of $\mathcal{B}$ of all fields containing $L$. Let $n(\mathcal{B})$ denote the greatest common divisor of the degrees $[E: F]$ over all fields $E \in \mathcal{B}$ of finite degree over $F$. (We set $n(\mathcal{B})=0$ if $\mathcal{B}$ has no finite degree extensions.) In particular, $n(\mathcal{A})=1$. If $F$ is $p$-special then $n(\mathcal{B})$ is a power of $p$ or 0 , and if $n(\mathcal{B})>0$ then there is a field $E \in \mathcal{B}$ such that $[E: F]=n(\mathcal{B})$.

For every $a \in F$, let $v_{a}$ be the discrete valuation of the rational function field $F(t)$ over $F$ corresponding to the irreducible polynomial $f(t)=t-a$.

Let $\mathcal{B} \subset \mathcal{A}$ be a subclass of field extensions of $F$. Two fields $L$ and $L^{\prime}$ in $\mathcal{B}$ of the same degree $n$ over $F$ are called simply $\mathcal{B}$-equivalent if there is a degree $n$ field extension $E / F(t)$ such that $E \in \mathcal{B}$ and two discrete valuations of $E$ over $v_{0}$ and $v_{1}$ with residue fields isomorphic to $L$ and $L^{\prime}$ respectively over $F$. Two fields $L$ and $L^{\prime}$ in $\mathcal{B}$ are called $\mathcal{B}$-equivalent if there is a chain of fields $L_{0}=L, L_{1}, \ldots, L_{r}=L^{\prime}$ in $\mathcal{B}$ such that $L_{i}$ is simply $\mathcal{B}$-equivalent to $L_{i+1}$ for all $i=0,1, \ldots, r-1$.

Note that if two fields $L$ and $L^{\prime}$ in $\mathcal{B}$ contain a common subfield $K \in \mathcal{A}$, i.e. $L, L^{\prime} \in \mathcal{B}_{K}$, then $L \sim_{\mathcal{B}_{K}} L^{\prime}$ implies $L \sim_{\mathcal{B}} L^{\prime}$

Let $X$ be a scheme of finite type over $F$. Denote by $\mathcal{A}(X)$ the class of all fields $E \in \mathcal{A}$ such that $X(E) \neq \emptyset$. The number $n(\mathcal{A}(X))$ coincides with $n(X)$, and $\mathcal{A}(X)$-equivalent fields will be called $X$-equivalent.

Example 6.1. In the notation of Proposition 4.5 the classes $\mathcal{A}\left(X_{S}\right)$ and $\mathcal{A}\left(X_{S^{\prime}}\right)$ coincide.

We shall need the following result.

Lemma 6.2. Let $X$ be a proper scheme of finite type over $F$ such that $\overline{\mathrm{CH}}_{0}\left(X_{L}\right)=0$ for any field $L \in \mathcal{A}(X)$ (e.g. $X$ is a projective homogeneous variety by Corollary 4.3). Let $x$ and $x^{\prime}$ be two closed points of $X$ such that residue fields $F(x)$ and $F\left(x^{\prime}\right)$ are isomorphic over $F$. Then $[x]=\left[x^{\prime}\right]$ in $\mathrm{CH}_{0}(X)$.

Proof. Let $L=F(x)$. By assumption there are rational points $y$ and $y^{\prime}$ of $X_{L}$ over $x$ and $x^{\prime}$ respectively. As $[y]=\left[y^{\prime}\right]$ in $\mathrm{CH}_{0}\left(X_{L}\right)$, taking the push-forward homomorphism with respect to $X_{L} \rightarrow X$ we get $[x]=f_{*}([y])=f_{*}\left(\left[y^{\prime}\right]\right)=\left[x^{\prime}\right]$.

Lemma 6.3. Let $X$ be a proper scheme of finite type over $F$ such that $\overline{\mathrm{CH}}_{0}\left(X_{L}\right)=0$ for any field $L \in \mathcal{A}(X)$. Let $x$ and $x^{\prime}$ be two closed points of $X$ of degree $n(X)$ such that residue fields $F(x)$ and $F\left(x^{\prime}\right)$ are $X$-equivalent. Then $[x]=\left[x^{\prime}\right]$ in $\mathrm{CH}_{0}(X)$.

Proof. We may assume that $F(x)$ and $F\left(x^{\prime}\right)$ are simply $X$-equivalent. Let $E$ be the field extension of $F(t)$ of degree $n=n(X)$ in the definition of the simple equivalence between $F(x)$ and $F\left(x^{\prime}\right)$. We have the dominant morphism $\operatorname{Spec} E \rightarrow \operatorname{Spec} F(t) \rightarrow \mathbb{P}_{F}^{1}$. Since $E \in \mathcal{A}(X)$, there is a morphism Spec $E \rightarrow X$ over $F$. Denote by $C$ the closure of the image of the diagonal morphism $\operatorname{Spec} E \rightarrow X \times \mathbb{P}_{F}^{1}$. Thus $C$ is an integral proper curve with a surjective morphism $f: C \rightarrow \mathbb{P}_{F}^{1}$. By assumption there are two discrete valuations $v$ and $v^{\prime}$ of $E$ over the valuations $v_{0}$ and $v_{1}$ of $F(t)$ respectively. Let $v$ and $v^{\prime}$ dominate points $c$ and $c^{\prime}$ on $C$ respectively, so that $f(c)=0$ and $f\left(c^{\prime}\right)=1$. Note that the residue field of $c$ is isomorphic to a subfield of the residue field of $v$, which in its turn is isomorphic to the field $F(x)$ of degree $n$ over $F$. On the other hand, $C \subset X \times \mathbb{P}_{F}^{1}$, 


\section{ZERO-CYCLES}

hence $n(C) \geqslant n(X)=n$. Therefore, $c$ is a closed point of degree $n, f^{-1}(0)=\operatorname{Spec} F(c)$ and $F(c) \simeq F(x)$. Similarly, $f^{-1}(1)=\operatorname{Spec} F\left(c^{\prime}\right)$ and $F\left(c^{\prime}\right) \simeq F\left(x^{\prime}\right)$.

The morphism $f$ is flat, hence $[c]=f^{*}([0])=f^{*}([1])=\left[c^{\prime}\right]$ in $\mathrm{CH}_{0}(C)$. Consider the projection $g: C \rightarrow X$. Let $z=g(c)$ and $z^{\prime}=g\left(c^{\prime}\right)$. As $F(z)$ is isomorphic to a subfield of $F(c)$ and $n(X)=n$, we have $F(z) \simeq F(c) \simeq F(x)$. Similarly $F\left(z^{\prime}\right) \simeq F\left(c^{\prime}\right) \simeq F\left(x^{\prime}\right)$. Therefore, $[z]=g_{*}([c])=g_{*}\left(\left[c^{\prime}\right]\right)=\left[z^{\prime}\right]$. By Lemma 6.2, $[x]=[z]=\left[z^{\prime}\right]=\left[x^{\prime}\right]$.

A class $\mathcal{B} \subset \mathcal{A}$ is called connected if for any special field $E \in \mathcal{A}$ every two fields in $\mathcal{B}_{E}$ of degree $n\left(\mathcal{B}_{E}\right)$ over $E$ are $\mathcal{B}_{E}$-equivalent. For example, the whole class $\mathcal{A}$ is connected, and therefore for every $X$ with $X(F) \neq 0$ the class $\mathcal{A}(X)=\mathcal{A}$ is connected.

Theorem 6.4. Let $X$ be a proper scheme of finite type over $F$ such that $\overline{\mathrm{CH}}_{0}\left(X_{L}\right)=0$ for any field $L \in \mathcal{A}(X)$. Suppose that the class $\mathcal{A}(X)$ is connected. Then $\overline{\mathrm{CH}}_{0}(X)=0$.

Proof. By Lemma 5.1 we may assume that $F$ is $p$-special for some prime $p$. It follows from Lemma 6.3 that, for any finite field extension $L / F$, every two closed points of $X_{L}$ of degree $n\left(X_{L}\right)$ are rationally equivalent. By Lemma 5.2, $\overline{\mathrm{CH}}_{0}(X)=0$.

\section{Severi-Brauer varieties}

Let $A$ be a central simple algebra of degree $m$ over a field $F$ and let $X$ be the corresponding SeveriBrauer variety of right ideals of $A$ of dimension $m$ (see [KMRT98, $\S 1$ ). The variety $X$ is projective homogeneous of the group $\mathbf{S L}_{1}(A)$ of type $S=\left\{\alpha_{2}, \ldots, \alpha_{m-1}\right\}$ where simple roots $\alpha_{1}, \ldots, \alpha_{m-1}$ of the Dynkin diagram $A_{m-1}$ are numbered as follows:

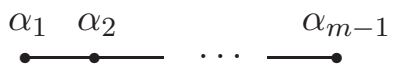

Here $m-1$ is the rank of $\mathbf{S L}_{1}(A)$. A field extension $L / F$ belongs to $\mathcal{A}(X)$ if and only if the algebra $A_{L}=A \otimes_{F} L$ is split. In particular $n(X)=\operatorname{ind}(A)$. If $A$ is split, i.e. $A=\operatorname{End}(V)$ for some vector space $V$, the Severi-Brauer variety $X$ is the projective space $\mathbb{P}(V)$.

Let $D$ be a (unique up to isomorphism) central division algebra $F$-algebra Brauer equivalent to $A$. Every maximal subfield of $D$ has degree $n=n(X)$ over $F$ and it is a splitting field for $D$ and $A$ and it belongs to $\mathcal{A}(X)$. Conversely, every field in $\mathcal{A}(X)$ of the smallest degree $n$ is isomorphic over $F$ to a maximal subfield of $D$ [Her94, $\S 4.4]$.

For every element $d \in D$ denote by $\operatorname{Prd}_{d}=\operatorname{Prd}_{d}(s) \in F[s]$ the reduced characteristic polynomial of $d$. The polynomial $\operatorname{Prd}_{d}$ is monic of degree $n$ and $\operatorname{Prd}_{d}$ is irreducible if and only if $d$ generates a maximal subfield of $D$. In this case $F(d) \simeq F[s] /\left(\operatorname{Prd}_{d}\right)$.

Recall that a finite field extension $L / F$ is called simple if $L$ is generated over $F$ by one element. In particular every separable finite extension is simple.

Lemma 7.1. Let $L \subset D$ be a simple maximal subfield and let $d \in D$. Then there is a maximal subfield $K \subset D$ containing $d$ such that $L \sim_{X} K$.

Proof. We have $L=F(a)$ for some $a \in D$. In particular the polynomial $\operatorname{Prd}_{a}$ is irreducible. Consider the element $c=t d+(1-t) a \in D[t]$ and its reduced characteristic polynomial $\operatorname{Prd}_{c} \in F[s, t]$. Note that $\operatorname{Prd}_{c}(s, 0)=\operatorname{Prd}_{a}$ and $\operatorname{Prd}_{c}(s, 1)=\operatorname{Prd}_{d}$. In particular $\operatorname{Prd}_{c}$ is irreducible monic in $s$.

Set $R=F[s, t] /\left(\operatorname{Prd}_{c}\right)$. Since $\operatorname{Prd}_{c}$ is irreducible, $R$ is a domain. The quotient field $E$ of $R$ is the maximal subfield of $D_{F(t)}$ generated by $c$ and therefore belongs to $\mathcal{A}(X)$. Let $Q$ be the principal 


\section{Chernousov and A. Merkurjev}

ideal of $R$ generated by $t$. The factor $\operatorname{ring} R / Q$ is isomorphic to $F[s] /\left(\operatorname{Prd}_{a}\right) \simeq L$. In particular, $Q$ is a maximal ideal of $R$. As the localization $R_{Q}$ is a Noetherian local domain of dimension 1 with principal maximal ideal, $R_{Q}$ is a discrete valuation ring. The corresponding discrete valuation of $E$ with residue field $L$ restricts to $v_{0}$ on $F(t)$.

Let $v$ be a discrete valuation of $E$ restricting to $v_{1}$. Since $R$ is integral over $F[t], R$ is contained in the valuation ring $O$ of $v$. As $R /(1-t)=F[s] /\left(\operatorname{Prd}_{d}\right)$ and $\operatorname{Prd}_{d}$ is a power of the minimal polynomial $h=h(s)$ of $d$, the intersection of $R$ with the maximal ideal of $O$ is generated by $1-t$ and $h$. In particular residue field $K$ of $O$ contains $F[s] /(h)=F(d)$.

On the other hand, since $X$ is proper, a morphism Spec $E \rightarrow X$ extends to $\operatorname{Spec} O \rightarrow X$. Therefore $K$ contains $F(x)$ for some $x \in X$. But $[K: F] \leqslant[E: F(t)]=n$, therefore $K=F(x)$ and $[K: F]=n$. Thus $K$ is isomorphic to a maximal subfield of $D$ containing $d$ and $L \sim_{X} K$.

Theorem 7.2. Let $A$ be a central simple $F$-algebra and let $X$ be the Severi-Brauer variety $S B(A)$. Then the class $\mathcal{A}(X)$ is connected.

Proof. It is sufficient to show that every two maximal subfields of $D$ are $X$-equivalent. We proceed by induction on $\operatorname{deg} D$ and may assume that $\operatorname{deg}(D)>1$. Choose a separable maximal subfield $L$ of $D$. It is sufficient to show that $L$ is $X$-equivalent to any maximal subfield $E \subset D$. Choose an element $d \in E \backslash F$. By Lemma 7.1, $L \sim_{X} K$ for some maximal subfield $K$ containing $d$. Let $M=F(d)$ and consider the centralizer $D^{\prime}$ of $M$ in $D$. The fields $E$ and $K$ are maximal subfields of $D^{\prime}$. By the induction hypothesis, $K \sim_{X_{M}} E$ and therefore $K \sim_{X} E$. Finally, $L \sim_{X} K \sim_{X} E$.

Theorem 6.4 yields the following corollary.

Corollary 7.3 (cf. [Pan84]). Let $X$ be a Severi-Brauer variety. Then $\overline{\mathrm{CH}}_{0}(X)=0$.

\section{Generalized Severi-Brauer varieties}

Let $A$ be a central simple $F$-algebra of degree $m$. Denote by $X=S B(2, A)$ the generalized SeveriBrauer variety of right ideals of $A$ of dimension $2 m$ (see [KMRT98, $\S 1]$ ). It is a projective homogeneous variety of the algebraic group $\mathbf{S L}_{1}(A)$ of type $S=\left\{\alpha_{1}, \alpha_{3}, \ldots, \alpha_{m-1}\right\}$ where simple roots are numbered as in $\S 7$. A field extension $L / F$ belongs to $\mathcal{A}(X)$ if and only if ind $A_{L} \leqslant 2$. If $A$ is split, i.e. $A=\operatorname{End}(V)$ for some vector space $V$, the variety $S B(2, A)$ is the Grassmannian variety of planes in $V$.

If the index of $A$ is odd, then a field extension $L / F$ belongs to $\mathcal{A}(X)$ if and only if $L$ splits $A$. Therefore the class $\mathcal{A}(X)$ coincides with $\mathcal{A}(S B(A))$. Hence the class $\mathcal{A}(X)$ is connected, by Theorem 7.2.

Suppose now that ind $A=2 n$ is even. We have $n(X)=n$. Let $D$ be a central division $F$-algebra of degree $2 n$ Brauer equivalent to $A$. In particular $\mathcal{A}(X)=\mathcal{A}(S B(2, D))$. Every finite extension of $F$ of degree $n$ in $\mathcal{A}(X)$ is isomorphic to a subfield of $D$ and conversely every subfield of $D$ of degree $n$ is contained in $\mathcal{A}(X)$.

Suppose that $D$ has a symplectic involution $\sigma$. Denote by $\operatorname{Symd}(D, \sigma)$ the subspace of $D$ of all elements $d+\sigma(d)$ for $d \in D$. The characteristic polynomial of an element $a \in \operatorname{Symd}(D, \sigma)$ is the square of the monic pfaffian characteristic polynomial $\operatorname{Prp}_{a}(s)$ of degree $n$ (see [KMRT98, Proposition 2.9]). In particular, $[F(a): F] \leqslant n$; moreover the polynomial $\operatorname{Prp}_{a}(s)$ is irreducible if and only if $[F(a): F]=n$.

Lemma 8.1. For any subfield $K$ of $D$ of degree $n$ there is a symplectic involution $\tau$ of $D$ such that $K \subset \operatorname{Symd}(D, \tau)$. 


\section{ZERO-CYCLES}

Proof. The centralizer $Q$ of $K$ in $D$ is a quaternion algebra over $K$. By [KMRT98, Theorem 4.14], the canonical involution of $Q$ can be extended to a symplectic involution $\tau$ on $D$. Choose an element $y \in Q$ such that $y+\tau(y)=1$. Then $x=x y+\tau(x y)$ for every $x \in K$, i.e. $K \subset \operatorname{Symd}(D, \tau)$.

Lemma 8.2. Let $\sigma$ and $\tau$ be two distinct symplectic involutions on $D$ and let $L$ be a simple subfield of $\operatorname{Symd}(D, \sigma)$ of degree $n$. Then the following hold.

(i) There exists a subfield $K$ of $D$ of degree $n$ such that $L \sim_{X} K$ and $K \cap \operatorname{Symd}(D, \tau)$ contains a nonconstant element.

(ii) For every $d \in \operatorname{Symd}(D, \sigma)$ there exists a subfield $K$ of $D$ of degree $n$ such that $d \in K$ and $L \sim_{X} K$.

Proof. Let $L=F(a)$ for some $a \in \operatorname{Symd}(D, \sigma)$.

(1) Choose $b \in D^{\times}$such that $\sigma=\operatorname{Int}(b) \circ \tau$ [KMRT98, Proposition 2.7]. We have $b a \in$ $\operatorname{Symd}(D, \tau)$. Replacing $a$ by $a+1$ if necessary we assume that $b a \neq F$.

Consider the element $c=t+(1-t) b \in D[t]$ and the involution $\sigma_{t}=\operatorname{Int}(c) \circ \sigma_{F(t)}$ on $D_{F(t)}$. Then $c a \in \operatorname{Symd}\left(D_{F(t)}, \sigma_{t}\right)$. The pfaffian characteristic polynomial $\operatorname{Prp}_{c a}$ belongs to $F[s, t]$. We have $\operatorname{Prp}_{c a}(s, 0)=\operatorname{Prp}_{b a}$ and $\operatorname{Prp}_{c a}(s, 1)=\operatorname{Prp}_{a}$; in particular $\operatorname{Prp}_{c a}$ is irreducible. Considering the quotient field $E$ of the factor ring $F[s, t] /\left(\operatorname{Prp}_{c a}\right)$ as in the proof of Lemma 7.1, we find two discrete valuations of $E$ over $v_{1}$ and $v_{0}$ respectively with residue fields isomorphic to $L$ and a subfield $K$ of $D$ containing the nonconstant element $b a$. Thus $L \sim_{X} K$.

(2) Let $E$ be the degree $n$ subfield of $D_{F(t)}$ generated by $t a+(1-t) d$. As in the proof of Lemma 7.1 we see that there are two discrete valuations of $E$ with residue fields $L=F(a)$ and $K$ respectively, where $K$ is a subfield of $D$ of degree $n$ and $d \in K$.

Theorem 8.3. Let $X$ be the generalized Severi-Brauer variety $S B(2, A)$ of a central simple algebra $A$ of exponent not divisible by 4 . Then the class $\mathcal{A}(X)$ is connected.

Proof. By definition of connectedness we may assume that $F$ is a $p$-special field. If $p$ is odd we already know that the class $\mathcal{A}(X)$ is connected. Thus we may assume that $p=2, \exp (A)=2$ and $\operatorname{ind}(A)=2 n$ is even. By [KMRT98, Theorem 3.8], the algebra $A$ has a symplectic involution.

We use the notation above. It is sufficient to show that every two subfields of $D$ of degree $n$ are $X$-equivalent.

Let $L$ and $E$ be subfields of $D$ of degree $n$. We may assume that $L / F$ is separable (see [Tit68, Proposition 5]) and therefore is simple. We prove that $L \sim_{X} E$ by induction on $n$. By Lemma 8.1, we can choose symplectic involutions $\sigma$ and $\tau$ of $D$ such that $L \subset \operatorname{Symd}(A, \sigma)$ and $E \subset \operatorname{Symd}(A, \tau)$.

Case 1: $\sigma=\tau$. Choose an element $d \in E \backslash F$. By Lemma 8.2(2), there is a subfield $K$ of $D$ of degree $n$ containing $d$ such that $L \sim_{X} K$. Let $D^{\prime}$ be the centralizer of $M=F(d)$ in $D$. By the induction hypothesis applied to $D^{\prime}$ with the involution $\left.\tau\right|_{D^{\prime}}$ we have $K \sim_{X_{M}} E$, therefore $K \sim_{X} E$ and $L \sim_{X} K \sim_{X} E$.

Case 2: $\sigma \neq \tau$. By Lemma 8.2(1), there exists a subfield $K$ of $D$ of degree $n$ such that $L \sim_{X} K$ and $K \cap \operatorname{Symd}(D, \tau)$ contains a nonconstant element $d$. Choose a subfield $H \subset \operatorname{Symd}(D, \tau)$ of degree $n$ containing $d$. Let $D^{\prime}$ be the centralizer of $M=F(d)$ in $D$. By the induction hypothesis applied to $D^{\prime}, K \sim_{X_{M}} H$ and therefore $K \sim_{X} H$. By the first case, $H \sim_{X} E$, hence $L \sim_{X} K \sim_{X} H \sim_{X} E$.

Corollary 8.4 (cf. [Kra05]). Let $X$ be the generalized Severi-Brauer variety $S B(2, A)$ of a central simple algebra $A$ of exponent not divisible by 4 . Then $\overline{\mathrm{CH}}_{0}(X)=0$. 


\section{Chernousov and A. Merkurjev}

\section{Quadrics}

Let $q$ be a quadratic form on an $F$-vector space $V$ of finite dimension. Denote by $X$ the projective quadric hypersurface in $\mathbb{P}(V)$ defined by the equation $q=0$. If $X$ is smooth, it is a projective homogeneous variety of the special orthogonal group $\mathbf{O}^{+}(q)$ of type $S=\left\{\alpha_{2}, \ldots, \alpha_{m}\right\}$ where simple roots $\alpha_{1}, \ldots \alpha_{m}$ of the Dynkin diagrams $D_{m}$ and $B_{m}$ are numbered as follows:
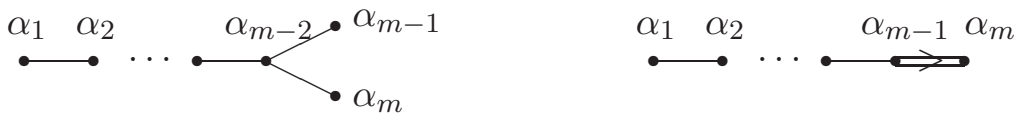

Here the first and second diagrams correspond to the cases $\operatorname{dim} V=2 m$ and $\operatorname{dim} V=2 m+1$ respectively.

The quadric $X$ has a rational point if and only if $q$ is isotropic. If $q$ is anisotropic, then $n(X)=2$ by Springer's theorem.

Example 9.1. Let $q$ be a nonzero two-dimensional quadratic form. Then we have $\operatorname{dim} X=0$; moreover $X=\operatorname{Spec} C_{0}(q)$; where $C_{0}(q)$ is the even Clifford algebra of $q$. The form $q$ is nondegenerate (respectively anisotropic) if and only if $C_{0}(q)$ is an étale quadratic $F$-algebra (respectively a quadratic field extension of $F$ ).

Theorem 9.2. The class $\mathcal{A}(X)$ for a projective quadric $X$ is connected.

Proof. We may assume that $q$ is anisotropic. Let $L / F$ be a quadratic field extension making $q$ isotropic and let $x \in X$ be a point with $F(x) \simeq L$. Let $\{1, \alpha\}$ be a basis of $L$ over $F$ and let $w=u+\alpha v, u, v \in V$, be an isotropic vector in $V_{L}$. Then $w \in U_{L}$, where $U$ is the two-dimensional subspace of $V$ spanned by $v$ and $u$. Moreover, by Example 9.1, $F(x) \simeq C_{0}\left(\left.q\right|_{U}\right)$.

Conversely, if $U \subset V$ is a two-dimensional subspace, then

$$
X \cap \mathbb{P}(U)=\operatorname{Spec} F(x) \simeq \operatorname{Spec} C_{0}\left(\left.q\right|_{U}\right),
$$

where $x$ is a closed point of degree 2 . Thus it is sufficient to show that for every two two-dimensional subspaces $U$ and $U^{\prime}$, the quadratic field extensions $L=C_{0}\left(\left.q\right|_{U}\right)$ and $L^{\prime}=C_{0}\left(\left.q\right|_{U^{\prime}}\right)$ are $X$-equivalent. We may assume that $U \cap U^{\prime} \neq 0$.

Let $\{v, u\}$ and $\left\{v, u^{\prime}\right\}$ be bases of $U$ and $U^{\prime}$ respectively. Consider the $F[t]$-submodule $W$ of $V_{F[t]}$ generated by $v$ and $t u+(1-t) u^{\prime}$. Let $R=C_{0}\left(\left.q_{F[t]}\right|_{W}\right)$. The quotient field $E$ of $R$ belongs to $\mathcal{A}(X)$. Since $R /(t)=L^{\prime}$ is a field, the principal ideal $Q$ generated by $t$ is maximal. Therefore the localization $R_{Q}$ is a Noetherian local domain of dimension 1 with principal maximal ideal, i.e. $R_{Q}$ is a discrete valuation ring. The corresponding discrete valuation of $E$ with residue field $L^{\prime}$ restricts to $v_{0}$ on $F(t)$. Similarly, there is a discrete valuation of $E$ with residue field $L$ restricting to $v_{1}$, i.e. $L$ and $L^{\prime}$ are simply $X$-equivalent.

Corollary 9.3 (cf. [Kar91, Swa89]). Let $X$ be a smooth projective quadric. Then $\overline{\mathrm{CH}}_{0}(X)=0$.

\section{Involution varieties}

Let $A$ be a central simple algebra of degree $2 m$ with a quadratic pair $(\sigma, f)$, where $\sigma$ is an involution on $A$ of the first kind and $f: \operatorname{Sym}(A, \sigma) \rightarrow F$ is a linear map satisfying certain properties [KMRT98, $\S 5$.B]. If $A$ is split, i.e. $A \simeq \operatorname{End}(V)$ for a $2 m$-dimensional vector space $V$ over $F$, the space $\operatorname{Sym}(A, \sigma)$ is identified with the subspace of symmetric elements in $V \otimes_{F} V$ and $f(v \otimes v)=q(v)$ for a nondegenerate quadratic form $q$ on $V$. 


\section{ZERO-CYCLES}

Consider the morphism

$$
\varphi: S B(A) \rightarrow \mathbb{P}(\operatorname{Sym}(A, \sigma))
$$

taking an ideal $I$ in $A$ to the (one-dimensional) subspace $I \cdot \sigma(I)$ of $\operatorname{Sym}(A, \sigma)$ [KMRT98, p. 122]. Let $W=\operatorname{Ker}(f)$. The inverse image $X=\varphi^{-1} \mathbb{P}(W)$ is called the involution variety of the quadratic pair. Thus $X$ is a hypersurface in the Severi-Brauer variety $S B(A)$. In the split case as above, $X$ is a smooth projective quadric hypersurface in $\mathbb{P}(V)$ given by the quadratic form $q$. In other words, $X$ is a twisted form of a projective quadric. It is a projective homogeneous variety of the special orthogonal group $\mathbf{O}^{+}(A, \sigma, f)$ (see [KMRT98, $\left.\S 26\right]$ ) of type $S=\left\{\alpha_{2}, \ldots, \alpha_{m}\right\}$, where $S$ is the subset of vertices of the Dynkin diagram $D_{m}$. In fact, any twisted form of a smooth projective quadric is isomorphic to an involution variety.

If $A$ is not split, then ind $A$ is even. Consider the case $\operatorname{ind}(A)=2$. Let $Q$ be a quaternion division algebra Brauer equivalent to $A$. Then $A \simeq \operatorname{End}_{Q}(V)$ for some right $Q$-module $V$. The Severi-Brauer variety $S B(A)$ can be identified with the variety $\mathbb{P}_{Q}(V)$ of reduced rank $1 Q$-submodules in $V$. Note that for any $Q$-submodule $U \subset V$ of rank 1 , the subvariety $\mathbb{P}_{Q}(U)$ of $\mathbb{P}_{Q}(V)$ is a conic curve. Then either the conic $\mathbb{P}_{Q}(U)$ is contained in $X$ or the intersection of $\mathbb{P}_{Q}(U)$ with $X$ is a point of degree 2. Therefore $n(X)=2$.

Theorem 10.1. If $\operatorname{ind}(A)=2$ then the class $\mathcal{A}(X)$ is connected.

Proof. As $n(X)=2$ it is sufficient to show that every two closed points $x$ and $x^{\prime}$ of degree 2 in $X$ are $X$-equivalent. By [Mer95b, Lemma 1], there are $Q$-submodules $U \subset V$ and $U^{\prime} \subset V$ of rank 1 such that $x \in \mathbb{P}_{Q}(U)$ and $x^{\prime} \in \mathbb{P}_{Q}\left(U^{\prime}\right)$. In fact $F(x)$ and $F\left(x^{\prime}\right)$ are isomorphic to the even Clifford algebras of the restrictions of the quadratic pair to $U$ and $U^{\prime}$ respectively. Let $v$ and $v^{\prime}$ be nonzero vectors of $U$ and $U^{\prime}$ respectively. Consider $w=t v+(1-t) v^{\prime} \in V_{F[t]}$ and let $W=Q[t] w$. Let $R$ be the even Clifford algebra of the restriction of the quadratic pair $(\sigma, f) \otimes F[t]$ on $W$. As in the proof of Theorem 9.2 we see that the quotient field $E$ of $R$ is a quadratic extension of $F(t)$ equipped with two discrete valuations over $v_{0}$ and $v_{1}$ respectively and residue fields isomorphic to $F(x)$ and $F\left(x^{\prime}\right)$, i.e. $F(x)$ and $F\left(x^{\prime}\right)$ are simply $X$-equivalent.

Consider the general case when $\operatorname{ind}(A)=2 n$. Assume that $F$ is a 2 -special field. We claim that $n(X)=2 n$. Indeed, $n(X) \geqslant 2 n$ since $X$ is a hypersurface in $S B(A)$ and $n(S B(A))=2 n$. On the other hand, choose a field extension $K / F$ of degree $n$ such that ind $A_{K}=2$. As $n\left(X_{K}\right)=2$, we conclude that $n(X) \leqslant 2 n$.

Set $Y=S B(2, A)$. We know that $n(Y)=n$.

Lemma 10.2. Let $y_{0}$ and $y_{1}$ be two closed points of $Y$ of degree $n$ such that $F\left(y_{0}\right)$ and $F\left(y_{1}\right)$ are simply $Y$-equivalent. Then there exist points $x_{0}$ and $x_{1}$ of $X$ of degree $2 n$ such that $F\left(x_{0}\right)$ and $F\left(x_{1}\right)$ are simply $X$-equivalent and $F\left(y_{i}\right)$ is isomorphic to a subfield of $F\left(x_{i}\right)$ for $i=0,1$.

Proof. Let $E / F(t)$ be an extension of degree $n$ such that $Y(E) \neq \emptyset$ and let $w_{i}, i=0,1$, be two discrete valuations of $E$ over the valuations $v_{i}$ of $F(t)$ with $F\left(w_{i}\right) \simeq F\left(y_{i}\right)$.

Since ind $A_{E}=2$, there is a quadratic field extension $K / E$ such that $X(K) \neq \emptyset$. Let $u_{i}$ be a discrete valuation of $K$ extending $w_{i}$ and let $L_{i}$ be the residue field of $u_{i}$. As $X$ is projective, a morphism Spec $K \rightarrow X$ factors through a morphism Spec $O_{i} \rightarrow X$, where $O_{i}$ is the valuation ring of $u_{i}$. In particular $X\left(L_{i}\right) \neq \emptyset$, therefore

$$
2 n=[K: F(t)] \geqslant\left[L_{i}: F\right] \geqslant n(X)=2 n .
$$

Hence there is a closed point $x_{i} \in X$ of degree $2 n$ with $F\left(x_{i}\right) \simeq L_{i}$. Note that $L_{0}$ and $L_{1}$ are simply $X$-equivalent and $F\left(y_{i}\right) \simeq F\left(w_{i}\right) \subset L_{i}$. 


\section{Chernousov and A. Merkurjev}

Theorem 10.3. Let $A$ be a central simple algebra of even degree with a quadratic pair and let $X$ be the corresponding involution variety. Then the class $\mathcal{A}(X)$ is connected.

Proof. We may assume that $F$ is $p$-special. If $p \neq 2$, then $X(F) \neq \emptyset$ and the class $\mathcal{A}(X)$ is connected. Let $F$ be a 2 -special field. If $A$ splits, then $X$ is a smooth projective quadric, and the result follows from Theorem 9.2. Thus we may assume that ind $A=2 n$ for some $n \geqslant 1$.

Let $x$ and $x^{\prime}$ be two closed points of $X$ of degree $2 n$. We would like to show that $F(x)$ and $F\left(x^{\prime}\right)$ are $X$-equivalent. As $F$ is 2-special, we can choose closed points $y$ and $y^{\prime} \in Y$ of degree $n$ such that $F(y) \subset F(x)$ and $F\left(y^{\prime}\right) \subset F\left(x^{\prime}\right)$. By Theorem 8.3, $F(y)$ and $F\left(y^{\prime}\right)$ are $Y$-equivalent, i.e. there are closed points $y_{0}=y, y_{1}, \ldots, y_{r}=y^{\prime}$ of degree $n$ such that $F\left(y_{i}\right)$ and $F\left(y_{i+1}\right)$ are simply $Y$-equivalent for $i=0, \ldots, r-1$. By Lemma 10.2 applied to each pair $\left(y_{i}, y_{i+1}\right)$ there are closed points $x_{i}^{\prime}, x_{i+1}$ of $X$ of degree $2 n$ such that $F\left(x_{i}^{\prime}\right)$ and $F\left(x_{i+1}\right)$ are simply $X$-equivalent and $F\left(y_{i}\right) \subset F\left(x_{i}^{\prime}\right), F\left(y_{i+1}\right) \subset F\left(x_{i+1}\right)$.

For convenience set $x_{0}=x$ and $x_{r}^{\prime}=x^{\prime}$. For every $i=0, \ldots, r$, the fields $F\left(x_{i}\right)$ and $F\left(x_{i}^{\prime}\right)$ contain $F\left(y_{i}\right)$. The index of $A$ over $F\left(y_{i}\right)$ is equal to 2 . By Theorem 10.1, the fields $F\left(x_{i}\right)$ and $F\left(x_{i}^{\prime}\right)$ are $X_{F\left(y_{i}\right)}$-equivalent, and therefore are $X$-equivalent. Finally we have

$$
F(x)=F\left(x_{0}\right) \sim F\left(x_{0}^{\prime}\right) \sim F\left(x_{1}\right) \sim F\left(x_{1}^{\prime}\right) \sim \cdots \sim F\left(x_{r}\right) \sim F\left(x_{r}^{\prime}\right)=F\left(x^{\prime}\right) .
$$

Corollary 10.4 (cf. [Kra05]). Let $A$ be a central simple algebra of even degree with a quadratic pair and let $X$ be the corresponding involution variety. Then $\overline{\mathrm{CH}}_{0}(X)=0$.

\section{Cayley-Dickson and Jordan algebras}

Let $C$ be a Cayley-Dickson algebra over $F$. Denote by $N$ the norm form of $C$ given by $N(x)=$ $x \bar{x}$, where $x \mapsto \bar{x}$ is the canonical involution of $C$. It is known [KMRT98, Proposition 33.18, Theorem 33.19] that $N$ is a 3-fold Pfister form, and $C$ is split if and only if $N$ is split. Therefore, Theorem 9.2 applied to the quadric given by $N$ yields the following result.

THEOREM 11.1. The class of splitting fields of $C$ is connected.

Remark 11.2. One can give a direct proof of Theorem 11.1 along the lines of the proof of Lemma 7.1 using the fact that a quadratic field extension of $L / F$ splits $C$ if and only if $L$ can be embedded into $C$.

Let $J$ be an exceptional simple 27-dimensional Jordan algebra over a field $F$ of degree 3 arising from the first Tits construction [Jac68, ch. IX, § 12]. Recall that there is an associated central simple $F$-algebra $A$ of degree 3 and an element $a \in F^{\times}$. The algebra $J$ is split if and only if $a$ is a reduced norm for $A$.

Theorem 11.3. Let $J$ be an exceptional simple 27-dimensional Jordan algebra over a 3-special field $F$ of degree 3 arising from the first Tits construction. Then the class of all splitting fields of $J$ is connected.

Proof. We may assume that $J$ is not split. Therefore for the class $\mathcal{B}$ of all splitting fields of $J$ we have $n(\mathcal{B})=3$. Let $L$ and $L^{\prime}$ be two cubic extensions of $F$ splitting $J$. We shall show that $L \sim_{\mathcal{B}} L^{\prime}$. By [PR84, Corollary 3] we may assume that $L$ and $L^{\prime}$ are subfields of $J$. Choose generators $x$ and $x^{\prime}$ of $L$ and $L^{\prime}$ over $F$ respectively and let $E$ be the subfield of $J_{F(t)}$ generated by $t x+(1-t) x^{\prime}$. As in the proof of Lemma 7.1 one shows using the cubic minimal polynomials of $x$ and $x^{\prime}$ that there are two discrete valuations of $E$ over $v_{0}$ and $v_{1}$ with residue fields $L$ and $L^{\prime}$ respectively, i.e. $L$ and $L^{\prime}$ are simply $\mathcal{B}$-equivalent. 


\section{ZERO-CYCLES}

\section{Groups of exceptional types}

In what follows $G_{0}$ denotes a split or quasi-split simple simply connected algebraic group over $F$ of one of the following types: $G_{2}, F_{4}, D_{4}, E_{6}, E_{7}$. Let $\xi \in Z^{1}\left(F, G_{0}\right)$ be a cocycle and let $G={ }^{\xi} G_{0}$ be the corresponding strongly inner form of $G_{0}$.

Theorem 12.1. Let $X=X_{S}$ be a projective homogeneous variety of the group $G$ as above. If $G$ is an outer form of type $E_{6}$ or of type $E_{7}$ we assume that $S \neq \emptyset$. If $G$ is a trialitarian group or of types $E_{6}, E_{7}$ we assume that $\operatorname{char}(F) \neq 2,3$. Then the class $\mathcal{A}(X)$ is connected. In particular, we have $\overline{\mathrm{CH}}_{0}(X)=0$.

Remark 12.2. If $G_{0}$ has type $E_{8}$ and $F$ is 5 -special, then the class $\mathcal{A}(X)$ is connected. This can be easily proven using the main result in [Che94] and Rost's chain lemma for symbols in $H^{3}\left(F, \mu_{5}\right)$. But we do not know whether this is still true for 2- and 3-special fields.

If $S=\Delta$, then $X_{S}=\operatorname{Spec} F$ and there is nothing to prove. Thus, by Proposition 4.5 we may assume that $S$ is a proper basic subset of $\Delta$. By Lemma 5.1, we may also assume that $F$ is $p$-special.

If $p \neq 2,3$, then we have $H^{1}\left(F, G_{0}\right)=1$ for all types under consideration. Indeed, by Steinberg's theorem [Ste65], $\xi$ is equivalent to a cocycle with coefficients in a maximal torus $T \subset G_{0}$ and, by [PR94, Proposition 6.21, p. 375], we have $H^{1}(F, T)=1$ for $p$-special fields with $p \neq 2,3$. It follows that $G$ is split or quasi-split and hence $X_{S}(F) \neq \emptyset$. Thus we may assume that $p=2,3$.

To finish the proof we shall either show directly that $\mathcal{A}(X)$ is connected or construct a projective homogeneous variety $Y$ (under some algebraic simple group) such that:

(i) the class $\mathcal{A}(Y)$ is connected;

(ii) $\mathcal{A}\left(X_{S}\right)=\mathcal{A}(Y)$.

We shall construct $Y$ using case-by-case consideration.

\section{Types $G_{2}$ and $F_{4}$}

By [Tit66], each isotropic group of type $G_{2}$ is split. Hence the only basic type for $G$ is $S=\emptyset$. Since $X_{\emptyset}$ is a variety of Borel subgroups of $G$, the class $\mathcal{A}\left(X_{\emptyset}\right)$ consists of all field extensions making $G$ split.

Recall that the correspondence $C \mapsto G=\operatorname{Aut}(C)$ gives rise to a bijection between the set of isomorphism classes of Cayley-Dickson algebras and the set of isomorphism classes of simple groups of type $G_{2}$ [KMRT98, Theorem 26.19]. In particular the classes of splitting fields for $C$ and $G$ coincide. The connectedness of $\mathcal{A}\left(X_{\emptyset}\right)$ follows from Theorem 11.1.

We pass to type $F_{4}$. According to [Tit66], if $G$ is isotropic over a field extension $L / F$ but not $L$-split, then its Tits index is of the following form:

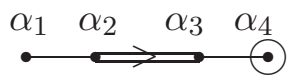

It follows that there exist two basic types only:

$$
S_{1}=\emptyset \quad \text { and } \quad S_{2}=\left\{\alpha_{1}, \alpha_{2}, \alpha_{3}\right\} .
$$

The class $\mathcal{A}\left(X_{S_{1}}\right)$ consists of all field extensions $L / F$ making $G$ split and $\mathcal{A}\left(X_{S_{2}}\right)$ consists of all field extensions $L / F$ making $G$ isotropic.

The correspondence $J \mapsto G=\operatorname{Aut}(J)$ gives rise to a bijection between the set of isomorphism classes of exceptional simple 27-dimensional Jordan algebra of degree 3 and the set of isomorphism classes of simple groups of type $F_{4}$ [KMRT98, Theorem 26.18]. In particular the classes of splitting fields for $J$ and $G$ coincide. 


\section{Chernousov and A. Merkurjev}

If $p=3$, then $J$ arises from the first Tits construction (cf. [Jac68, ch. IX, §12]). We claim that the diagram above cannot appear as the Tits index of $G$ over any extension $L / F$ of $F$. Indeed, assume the contrary. Since $G$ is not split over $L$, so is $J$. The anisotropic kernel of $G$ over $L$ has type $B_{3}$, hence there is a finite extension $E / L$ of degree $[E: L]=2^{a}$ for some positive integer $a$ making $G$ (and hence $J$ ) split. On the other side, the extension $E / L$ does not split $J$ since it arises from the first Tits construction, a contradiction. Thus we showed that the classes $\mathcal{A}\left(X_{S_{1}}\right), \mathcal{A}\left(X_{S_{2}}\right)$ and the class of all splitting fields of $J$ coincide and we are done by Theorem 11.3.

If $p=2$, then $J$ is reduced. Recall (cf. [GMS03, PR95]) that given $J$ one can associate a 3-Pfister form $f_{3}(J)$ and a 5-Pfister form $f_{5}(J)$ with the following properties (cf. [Spr60, Rac72]).

(a) The group $G$ is split over a field extension $L / F$ if and only if $f_{3}(J)$ is hyperbolic over $L$.

(b) The group $G$ is isotropic over a field extension $L / F$ if and only if $f_{5}(J)$ is hyperbolic over $L$.

Let $Y_{1}$ and $Y_{2}$ be the projective quadric defined by the equations $f_{3}(J)=0$ and $f_{5}(J)=0$ respectively. By (a) and (b), we have $\mathcal{A}\left(X_{S_{1}}\right)=\mathcal{A}\left(Y_{1}\right)$ and $\mathcal{A}\left(X_{S_{2}}\right)=\mathcal{A}\left(Y_{2}\right)$. The connectedness of $\mathcal{A}\left(X_{S_{1}}\right)$ and $\mathcal{A}\left(X_{S_{2}}\right)$ follows from Theorem 9.2.

\section{Type $D_{4}$}

Suppose first that $G$ is anisotropic of type ${ }^{1} D_{4}$ or ${ }^{2} D_{4}$. Then $G \simeq \operatorname{Spin}(f)$, where $f$ is an eightdimensional quadratic form with trivial even Clifford algebra. Let $d F^{\times 2}$ be the discriminant of $f$. The form $f \perp\langle 1,-d\rangle$ has trivial discriminant and Clifford algebra, and therefore it is represented by a 3 -Pfister form $g$ in the Witt ring $W(F)$.

As $g$ can have Witt index 0 or 4 over a field extension $L / F$, it follows that if $f$ becomes isotropic over $L / F$, then its Witt index over $L$ is either 1 or $f$ is quasi-split (i.e. its Witt index is either 3 or 4$)$. Hence for the Tits index of $G$ over $L$ there are the following possibilities only:
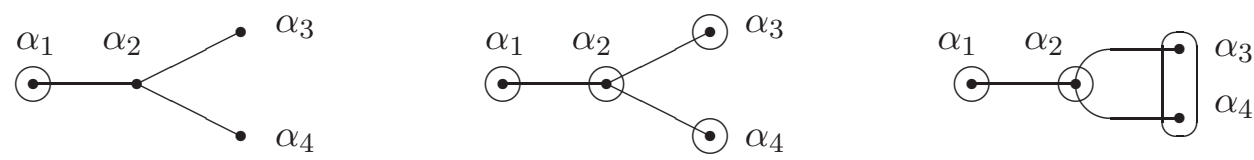

By Remark 4.6, we need to consider the following basic types only:

$$
S_{1}=\left\{\alpha_{2}, \alpha_{3}, \alpha_{4}\right\} \quad \text { and } \quad S_{2}=\emptyset .
$$

The variety $X_{S_{1}}$ is isomorphic to the quadric $Y$ defined by $f=0$. The class $\mathcal{A}(Y)$ is connected by Theorem 9.2.

The second case $S_{2}$ corresponds to the variety of Borel subgroups of $G$. A field extension $L / F$ makes $X_{\emptyset}$ isotropic if and only if it splits $g$. Let $Z$ be the projective quadric defined by $g=0$. Then the classes $\mathcal{A}\left(X_{\emptyset}\right)$ and $\mathcal{A}(Z)$ coincide, and we are done again by Theorem 9.2.

We now turn to trialitarian cases ${ }^{3} D_{4}$ and ${ }^{6} D_{4}$. We need to consider the case $p=3$ only. Let $E / F$ be a cubic extension over which $G$ is a classical group.

Lemma 14.1. Let $F$ be 3 -special. If $L / F$ is an extension such that $G$ is $L$-isotropic, then $G$ is quasi-split over $L$.

Proof. If $G$ is not quasi-split over $L$, then, by [Tit66], its Tits index over $L$ is of the following form:

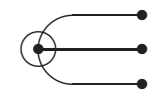




\section{ZERO-CYCLES}

Hence the semisimple $L$-anisotropic kernel of $G$ is isomorphic to $R_{E^{\prime} / L}\left(\mathbf{S L}_{1}(D)\right)$, where $D$ is a quaternion algebra over $E^{\prime}$ and $E^{\prime}=E \cdot L$. Note that such a group is still anisotropic over $E^{\prime}$. On the other side, since $F$ is 3 -special, $G$ is quasi-split over $E$ and hence over $E^{\prime}=E \cdot L$, a contradiction.

Lemma 14.1 implies that we need to consider the basic type $S=\emptyset$ only. Let $R_{G_{0}}: H^{1}\left(F, G_{0}\right) \rightarrow$ $H^{3}(F, \mathbb{Z} / 3)$ be the Rost invariant. We refer to [GMS03] for the definition and properties of the Rost invariant. It takes values in $\mathbb{Z} / 3$ since $F$ is 3 -special. By [Che03, Theorem 6.14], Ker $R_{G_{0}}=1$ and $R_{G_{0}}([\xi])$ is a 3 -symbol, say $(a) \cup(b) \cup(c)$, where $a, b, c \in F^{\times}$.

Let $J$ be the Jordan algebra arising from the first Tits construction corresponding to the central simple algebra $A=(a, b)$ over $F$ of degree 3 and element $c$. Recall that it is split over a field extension $L / F$ of $F$ if and only if $c \in \operatorname{Nrd} A_{L}$ or equivalently if and only if $R_{G_{0}}([\xi])$ is trivial over $L$, by [MS82, Theorem 12.2]. As $\operatorname{Ker} R_{G_{0}}=1$, the last holds if and only if $\left[\xi_{L}\right]=1$. Thus, by Lemma 3.2, the classes of splitting fields of $J$ and $\mathcal{A}\left(X_{\emptyset}\right)$ coincide and the connectedness of $X_{\emptyset}$ follows from Theorem 11.3.

\section{Inner forms of type ${ }^{1} E_{6}$}

According to [Tit66] all admissible Tits indices of type ${ }^{1} E_{6}$ corresponding to isotropic (not split) groups are as follows:

(a)

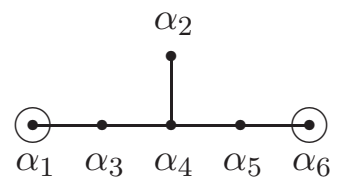

(b)

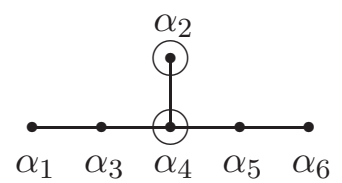

In case (b) the corresponding anisotropic kernel has type $A_{2}+A_{2}$. Since $\mathbf{S L}_{3} \times \mathbf{S L}_{3}$ has no nontrivial strongly inner forms, Theorem 3.3 implies that diagram (b) cannot appear as the Tits index of a strongly inner form of type ${ }^{1} E_{6}$ (note that in the notation of that theorem the condition $H^{1}(F, C / C \cap H)=1$ holds, since $C=C_{1}$ is an $F$-split torus). Thus, by Remark 4.6, the only basic types to be considered are

$$
S_{1}=\emptyset \quad \text { and } \quad S_{2}=\left\{\alpha_{2}, \alpha_{3}, \alpha_{4}, \alpha_{5}\right\} .
$$

Lemma 15.1. Assume that $G$ has diagram (a) over an extension $L / F$. Then the anisotropic $L$-kernel of $G$ is isomorphic to $\operatorname{Spin}(f)$ where $f$ is a 3-Pfister form over $L$.

Proof. The anisotropic $L$-kernel is a strongly inner form of type ${ }^{1} D_{4}$, by Theorem 3.3. So the result is clear.

Lemma 15.2. Let $F$ be 3 -special. Then $\mathcal{A}\left(X_{S_{1}}\right)=\mathcal{A}\left(X_{S_{2}}\right)$.

Proof. The inclusion $\mathcal{A}\left(X_{S_{1}}\right) \subset \mathcal{A}\left(X_{S_{2}}\right)$ is clear. Conversely, let $L \in \mathcal{A}\left(X_{S_{2}}\right)$. If $X_{S_{1}}(L)=\emptyset$, then $G$ has Tits index (a) over $L$. The corresponding anisotropic kernel of $G$ over $L$ is isomorphic to $\operatorname{Spin}(f)$ where $f$ is a 3 -Pfister form, by Lemma 15.1. Hence it can be split by a quadratic extension $E / L$. On the other side, the Rost invariant $R_{G_{0}}$ has trivial kernel (cf. [Che03, Gar01]), takes values in $H^{3}(F, \mathbb{Z} / 3)$ and $R_{G_{0}}([\xi])$ is a symbol, by [Che03]. Let $J$ be a Jordan algebra corresponding to the symbol $R_{G_{0}}([\xi])$. As Ker $R_{G_{0}}=1, J$ is nontrivial over $L$ and remains so over its quadratic extension $E / L$ since $J$ arises from the first Tits construction. However $J,[\xi]$ (and hence $G$ ) have the same splitting fields, a contradiction.

If $p=3$, then by Lemma $15.2, \mathcal{A}\left(X_{S_{1}}\right)=\mathcal{A}\left(X_{S_{2}}\right)$ and the classes of splitting fields of $J$ and $\mathcal{A}\left(X_{S_{1}}\right)$ coincide where $J$ is the Jordan algebra constructed in Lemma 15.2. The connectedness of $\mathcal{A}\left(X_{S_{1}}\right), \mathcal{A}\left(X_{S_{2}}\right)$ follows from Theorem 11.3. 


\section{Chernousov and A. Merkurjev}

Now, let $p=2$.

Lemma 15.3. If $F$ is 2-special, then $G$ is $F$-isotropic.

Proof. There exists the canonical embedding $H_{0} \rightarrow G_{0}$ where $H_{0}$ is a split group of type $F_{4}$. It induces the mapping $H^{1}\left(F, H_{0}\right) \rightarrow H^{1}\left(F, G_{0}\right)$ which is surjective, by [GMS03, page 51, Exercise 22.9] and [Gar01, Example 3.4], since $F$ is 2-special. Each class in $H^{1}\left(F, H_{0}\right)$ is split over a quadratic extension $E / F$, by property (a) in Section 13, hence so is [ $\xi$ ]. By [Che03, Lemma 6.4], $G$ is isotropic over $F$.

Lemma 15.3 implies that $X_{S_{2}}(F) \neq \emptyset$ and hence $\mathcal{A}\left(X_{S_{2}}\right)$ is connected. Furthermore, if $G$ is not split, then, by Lemma 15.1, its anisotropic kernel is isomorphic to $\mathbf{S p i n}(f)$ where $f$ is a 3-Pfister form $f$. Hence $\mathcal{A}\left(X_{S_{1}}\right)=\mathcal{A}(Y)$ where $Y$ is the quadric defined by $f=0$.

\section{Outer forms of type $E_{6}$}

If $p \neq 2$, then $G_{0}$ and $G$ are inner forms of type $E_{6}$. So we may assume that $p=2$. Then $F$ is perfect since by our assumption $\operatorname{char}(F) \neq 2$.

\subsection{Basic types}

Let $K=F(\sqrt{d})$ be the quadratic extension which makes $G_{0}$ (and hence $G$ ) an inner form of type $E_{6}$. We denote by $\sigma$ a unique nontrivial automorphism of $K / F$. According to Tits' classification [Tit66] if $G$ is $F$-isotropic but not quasi-split, then its $F$-index is one of the following:

(a)

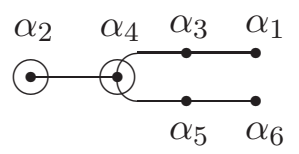

(c)

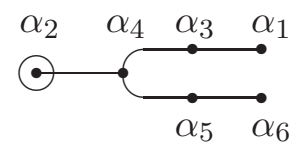

(b)

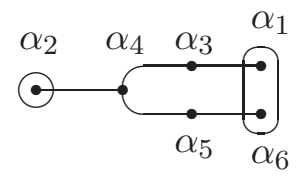

(d)

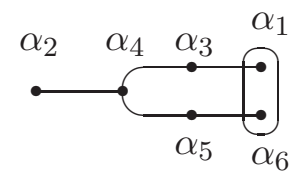

Lemma 16.1. The Tits index of $G$ is not of the form (a).

Proof. In case (a) the anisotropic kernel of $G$ has type $A_{2}+A_{2}$ and a maximal $F$-split torus $C_{1}$ has dimension 2. By dimension count $C_{1}$ coincides with the center $C$ of its centralizer; in particular $C$ and all its quotients have trivial cohomology in dimension 1. Hence, by Theorem 3.3, the anisotropic kernel of $G$ is a strongly inner form of $R_{K / F}\left(\mathbf{S L}_{3}\right)$. As $H^{1}\left(F, R_{K / F}\left(\mathbf{S L}_{3}\right)\right)=1$, the group $R_{K / F}\left(\mathbf{S L}_{3}\right)$ has no nontrivial strongly inner forms.

Thus there are four basic types only:

$$
S_{1}=\left\{\alpha_{2}, \alpha_{3}, \alpha_{4}, \alpha_{5}\right\}, \quad S_{2}=\left\{\alpha_{1}, \alpha_{3}, \alpha_{4}, \alpha_{5}, \alpha_{6}\right\}, \quad S_{3}=\left\{\alpha_{3}, \alpha_{4}, \alpha_{5}\right\}, \quad S_{4}=\emptyset .
$$

According to Lemma 15.3, $G$ is isotropic over $K$. If it is not $K$-split, then its Tits index is given by diagram (a) in Section 15 and hence its anisotropic kernel over $K$ is isomorphic to $\mathbf{S p i n}(f)$ where $f$ is a 3-Pfister form over $K$. Since $f$ can be split by a quadratic extension of $K$, we obtain that $n\left(X_{S_{4}}\right)$ divides 4 .

To compute the numbers $n\left(X_{S}\right)$ we need information on groups of type $D_{n}$. 


\section{ZERO-CYCLES}

\subsection{Classical groups of type $D_{n}$}

Recall that a simple simply connected $F$-group of type ${ }^{1} D_{n}$ can be realized as a spinor group $\operatorname{Spin}(A, \tau)$ associated to a central simple algebra $A$ over $F$ equipped with an involution $\tau$ of the first kind, orthogonal type and trivial discriminant. If $A$ is represented by a skew field $D$ in the Brauer group $\operatorname{Br}(F)$, then $A \simeq \operatorname{End}_{D}(V)$ where $V$ is a right $D$-module, and $\tau$ corresponds to a nondegenerate skew-hermitian form $h$ of trivial discriminant on $V$ with respect to a suitable involution $\tau^{\prime}$ on $D$ of symplectic type.

$\operatorname{Spin}(A, \tau)$ is isotropic over $F$ if and only if $h$ is isotropic, i.e. there is a vector $v \in V$ such that $h(v, v)=0$. The Tits index of $\operatorname{Spin}(A, \tau)$ over $F$ is of the following form:

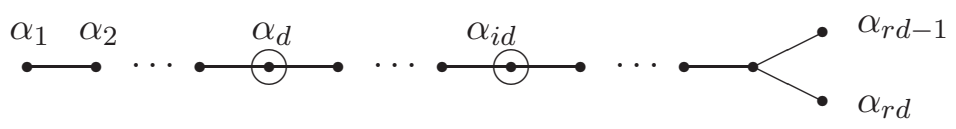

Here $d=\operatorname{deg}(D), i$ is the Witt index of $h$ and $r d=n$.

\subsection{Trialitarian effect}

We keep the above notation. Assume that we are given a pair $(A, \tau)$ such that $A$ has index 2 and degree 8. Consider the even Clifford algebra of $(A, \tau)$. It is of the form $A_{1} \times A_{2}$ where $A_{1}, A_{2}$ are central simple algebras of degree 8 equipped with involutions $\tau_{1}, \tau_{2}$ of orthogonal type and $A \cdot A_{1}$. $A_{2}=1$ in the Brauer group $\operatorname{Br}(F)$ (see [KMRT98]). The center $Z$ of $\operatorname{Spin}(A, \tau)$ is isomorphic to $Z \simeq \mathbb{Z} / 2 \times \mathbb{Z} / 2$. Three nontrivial elements in $Z$ give rise to the quotient morphisms $\phi_{i}: \operatorname{Spin}(A, \tau) \rightarrow$ $\mathbf{O}^{+}\left(A_{i}, \tau_{i}\right), i=1,2$ and $\phi: \operatorname{Spin}(A, \tau) \rightarrow \mathbf{O}^{+}(A, \tau)$. If $\mathbf{S p i n}(A, \tau)$ is isotropic over $F$, there are two possibilities for its Tits index:
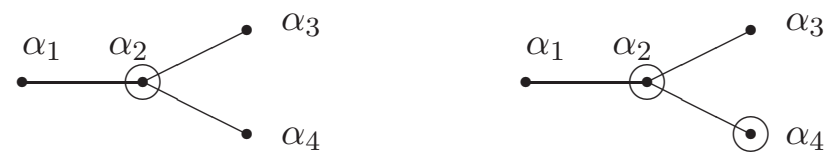

In the first case all algebras $A, A_{1}, A_{2}$ have index 2 , and in the second case one of the algebras $A_{1}, A_{2}$ is split and a quadratic form $f$ corresponding to its involution is isotropic. Note that according to the above picture isotropy of $f$ implies automatically that its Witt index is 2 .

Lemma 16.2. Assume that $A, A_{1}, A_{2}$ have indices at most 2. Then there is a quadratic extension $L / F$ such that $\operatorname{Spin}(A, \tau)$ has rank at least 2 over $L$.

Proof. If all algebras $A, A_{1}, A_{2}$ are matrix algebras, then $G \simeq \operatorname{Spin}(f)$ where $f$ is a 3-Pfister form. Such a form can be split by a quadratic extension.

Assume that $A$ has index 2. Consider the pair $\left(A_{1}, \tau_{1}\right)$ and the corresponding involution variety $Y$. As $n(Y)=2$, there is a quadratic extension $L / F$ belonging to $\mathcal{A}(Y)$. The remark above the lemma shows that the $L$-rank of $\operatorname{Spin}(A, \tau)$ is at least 2 .

\subsection{Computing $n\left(X_{S}\right)$}

Proposition 16.3. We have $n\left(X_{S_{1}}\right)=n\left(X_{S_{2}}\right)=n\left(X_{S_{3}}\right)=2$.

Proof. We consider the most difficult case when $G$ is anisotropic over $F$. Isotropic cases are reduced to groups of classical types and can be treated much more easily.

We may assume that $G$ is not split over $K$, since otherwise $X_{S_{i}}(K) \neq \emptyset, i=1, \ldots, 4$, and we are done. Then its Tits $K$-index is given by diagram (a) in Section 15 and so the variety of parabolic subgroups in $G$ of type $S_{1}=\left\{\alpha_{2}, \alpha_{3}, \alpha_{4}, \alpha_{5}\right\}$ contains a $K$-point. By Lemma 3.4 , there is a parabolic subgroup $P$ in $G$ over $K$ of type $S_{1}$ such that $H_{1}=P \cap \sigma(P)$ is a reductive part of $P$. 


\section{Chernousov and A. Merkurjev}

Now $H_{1}$ is defined over $F$ and is an almost direct product of a simple simply connected $F$-group of classical type $D_{4}$ and a two-dimensional central torus $T_{1}$. Since $T_{1}$ is $F$-anisotropic and split over $K, \sigma$ acts on the character lattice of $T_{1}$ by multiplication by -1 . Hence each 1-dimensional subtorus in $T_{1}$ is $F$-defined.

Let $\Sigma$ be a root system of $G$ with respect to an arbitrary maximal $F$-defined torus of $G$ containing $T_{1}$ and let $\tilde{\alpha}$ be the highest root in $\Sigma$ with respect to the ordering given on diagram (a) in Section 15 . The restriction mapping $\left.\tilde{\alpha}\right|_{T_{1}}: T_{1} \rightarrow G_{m}$ is a nontrivial homomorphism. Let $T_{2}$ be its kernel. It has dimension 1 and, by construction, $T_{2}$ commutes with $U_{ \pm \tilde{\alpha}}$. As $T_{2}$ is defined over $F$, so is $H_{2}=C_{G}\left(T_{2}\right)$, which is a reductive group whose semisimple part $\left[H_{2}, H_{2}\right]$ is a simple simply connected group of classical type $D_{5}$ generated by roots $\alpha_{2}, \alpha_{3}, \alpha_{4}, \alpha_{5}, \tilde{\alpha}$.

Let us describe the structure of $\left[H_{2}, H_{2}\right]$. Note first that it has $K$-rank 1 (and hence is $K$-isotropic), since $T_{1}$ is contained in $H_{2}=C_{G}\left(T_{2}\right)$ and $T_{1}$ is a maximal $K$-split torus in $G$ of dimension 2. Furthermore, up to isogeny, we have $\left[H_{2}, H_{2}\right] \simeq \mathbf{S U}(D, h)$ where $D$ is a skew field over $F$ equipped with a symplectic involution $\tau$ and $h$ is a skew-hermitian form over $D$ with respect to $\tau$. As $\left[H_{2}, H_{2}\right]$ is $K$-isotropic and its anisotropic semisimple kernel over $K$ coincides with that of $G$ (it is a group of type $D_{4}$ generated by $\left\{\alpha_{2}, \alpha_{3}, \alpha_{4}, \alpha_{5}\right\}$ ), Theorem 3.3 applied to $G$ over $K$ shows that this anisotropic kernel has trivial Tits algebras over $K$. It follows that the discriminant of $h$ is trivial over $K$, the algebra $D$ splits over $K$ and hence $D$ is either $F$ or a quaternion skew field over $F$.

We claim that the discriminant of $h$ is $d$. Indeed, we know that it is trivial over $K$, hence we need only to exclude the possibility for it to be trivial over $F$. Let $Z$ be the variety of Borel subgroups of $\left[H_{2}, H_{2}\right]$. If the discriminant of $h$ is trivial over $F$, then $\left[H_{2}, H_{2}\right]$ is a group of inner type and hence it has rank 5 over a field extension $F(Z) / F$. This implies automatically that $G$ is split over $F(Z)$. But $F$ is algebraically closed in $F(Z)$, hence $K$ is not contained in $F(Z)$. It follows that $G$ is still a group of outer type over $F(Z)$, a contradiction.

Assume now that $D$ is a quaternion skew field. By [Mer95b, Lemma 1], $h$ can be written in the form $h=h_{1} \oplus h_{2}$ where $h_{2}$ is a 1-dimensional skew-hermitian form over $D$ isotropic over $K$ and $h_{1}$ is a four-dimensional form with trivial discriminant. Lemma 16.2 applied to $\left(D, h_{1}\right)$ shows that there is a quadratic extension $L / F$ such that the $L$-rank of $\mathbf{S U}\left(D, h_{1}\right)$ is at least 2 . Note that the conditions of this lemma are satisfied since the anisotropic kernel of $\mathbf{S U}\left(D, h_{1}\right)$ over $K$ is a group of classical type $D_{4}$ with trivial Tits algebras. An inspection of possible Tits indices of $G$ over $L$ shows that $G$ is either quasi-split over $L$ or has Tits index (b) in the above list of Tits indices of outer type $E_{6}$. In all cases we have $X_{S_{1}}(L) \neq \emptyset, X_{S_{2}}(L) \neq \emptyset, X_{S_{3}}(L) \neq \emptyset$ and hence $n\left(X_{S_{1}}\right)=n\left(X_{S_{2}}\right)=n\left(X_{S_{3}}\right)=2$.

If $D=F$ and $h$ is a 10-dimensional quadratic form over $F$, we can apply the same argument as above. Namely, since $h$ is isotropic over $K$, we can write it in the form $h=h_{1} \oplus h_{2}$ where $h_{2}$ is a two-dimensional quadratic form splitting over $K$ and $h_{1}$ is an eight-dimensional quadratic form with trivial discriminant. Arguing as in Lemma 16.2 we easily see that $h_{1}$ has rank at least 2 over a proper quadratic extension $L / F$. So the result follows.

\subsection{Basic type $S_{1}$}

Let $L \in \mathcal{A}\left(X_{S_{1}}\right)$ and $[L: F]=2$. As $K \in \mathcal{A}\left(X_{S_{1}}\right)$, it suffices to show that $L$ and $K$ are $X_{S_{1}}$-equivalent. We shall construct an $F$-subgroup $H \subset G$ of classical type $D_{5}$ with the following properties:

(1) there is an $F$-embedding $Y \rightarrow X_{S_{1}}$, where $Y$ is the involution variety corresponding to $H$;

(2) $L, K \in \mathcal{A}(Y)$. 


\section{ZERO-CYCLES}

Property (2) implies that $L$ and $K$ are $Y$-equivalent, by Theorem 10.3, and property (1) implies that $L$ and $K$ are $X_{S_{1}}$-equivalent.

To construct $H$ with the required properties we repeat the argument in $\S 16.4$ verbatim. Namely, $G$ contains a parabolic subgroup $P$ of type $S_{1}$ over $L$. We may assume that $P$ is standard in the corresponding ordering of the root system of $G$. Let $\tau$ be the nontrivial automorphism of $L / F$. Without loss of generality we may assume that $H_{1}=P \cap \tau(P)$ is a reductive part of $P$, by Lemma 3.4. It is an $F$-reductive group whose semisimple part is a simple simply connected group of type $D_{4}$ generated by roots $\alpha_{2}, \alpha_{3}, \alpha_{4}, \alpha_{5}$ and whose central torus $T_{1}$ has dimension 2 . This torus is isotropic over $L$ and splits over $K \cdot L$.

Let $T_{2} \subset T_{1}$ be the kernel of the restriction mapping $\left.\tilde{\alpha}\right|_{T_{1}}$ where $\tilde{\alpha}$ is the highest root in the chosen ordering of the root system of $G$. Below we shall show that $T_{2}$ is $F$-defined. Then the centralizer $H_{2}=C_{G}\left(T_{2}\right)$ is a reductive $F$-group whose semisimple part $H=\left[H_{2}, H_{2}\right]$ is a simple simply connected group of type $D_{5}$ over $F$. We claim that it has the required properties.

Let us check property (1). The Dynkin diagram of $H$ is of the following form:

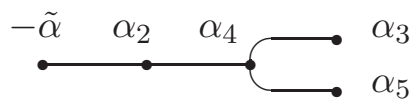

The corresponding involution variety $Y$ is the variety of parabolic subgroups in $H$ of type $S_{1}=$ $\left\{\alpha_{2}, \alpha_{4}, \alpha_{3}, \alpha_{5}\right\}$. The subgroup $P_{H}=P \cap H$ of $H$ is parabolic of type $S_{1}$. Then the required embedding is given by $H / P_{H} \rightarrow G / P, h P_{H} \rightarrow h P$. It is well defined and it is easy to check that it is $F$-defined using the twisted argument.

To check property (2) we need information about the structure of $T_{1}$. It is proven in [CT99, Theorem 2.12] that $T_{1}$ viewed over $L$ is isomorphic to $T_{1} \simeq R_{E / L}\left(\mathbf{G}_{m, E}\right)$ where $E=L \cdot K$. Thus $T_{1}$ viewed over $L$ is an almost direct product of a one-dimensional split $L$-subtorus, say $V_{1}$, and a one-dimensional $L$-anisotropic torus, $V_{2}=R_{E / L}^{(1)}\left(\mathbf{G}_{m, E}\right)$. It follows that $\operatorname{Gal}(L / F)$ preserves $V_{1}, V_{2}$ and hence both of them are $F$-defined. Note that computation in [CT99] shows that $V_{1}$ is contained in $H$ and commutes with the subgroup of $H$ generated by $\alpha_{2}, \alpha_{4}, \alpha_{3}, \alpha_{5}$. The last implies that $H$ is $L$-isotropic and $L \in \mathcal{A}(Y)$.

Lemma 16.4. We have $V_{2}=T_{2}$ and $T_{2}$ splits over $K$.

Proof. The 2-torsion part of $T_{1}$ is of the form $R_{E / L}\left(\mu_{2}\right)$. Using the same computations with the generators and relations of $G$ over $\bar{F}$ as in [CT99, Theorem 2.12] we easily find that the centralizer of $-1 \in R_{E / L}\left(\mu_{2}\right)$ in $G$ is $H \cdot T_{2}$, hence $T_{2}$ is $L$-defined. But $T_{1}$ contains only two $L$-defined subtori, namely $V_{1}$ and $V_{2}$. As $V_{1} \subset H$, we get $T_{2}=V_{2}$.

Assume that $T_{2}$ is not split over $K$. Let $Z$ be the variety $Z$ of Borel subgroups of $H$ over $K$. The group $H$ being quasi-split over $K(H / B)$ contains a split torus over $K(H / B)$, say $T_{3}$, of dimension at least 4 . One easily checks that $C_{G}\left(T_{3}\right)$ is a maximal torus in $G$ containing $T_{2}$. As $K$ is algebraically closed in $K(Z)$, the torus $T_{2}$ is still anisotropic over $K(Z)$ and hence $C_{G}\left(T_{3}\right)$ does not split over $K(Z)$.

On the other side, as the rank of $G$ over $K(H / B)$ is at least 4, Tits' classification implies that $G$ is split over $K(H / B)$ (see the diagrams in Section 15) and hence so is the torus $C_{G}\left(T_{3}\right)$ because any $K(Z)$-split torus in $G$ is contained in a maximal $K(Z)$-split torus of $G$. This contradicts our assumption that $T_{2}$ is anisotropic over $K(H / B)$.

As $T_{2}$ is $K$-split, the $K$-anisotropic kernel of $G$ is contained in $H_{2}=C_{G}\left(T_{2}\right)$, hence $G$ and $H$ have the same $K$-anisotropic kernel. Then Tits' classification of groups of inner type $E_{6}$ shows that $K \in \mathcal{A}(Y)$. 


\section{Chernousov and A. Merkurjev}

\subsection{Basic type $S_{2}$}

Assume that $L \in \mathcal{A}\left(X_{S_{2}}\right)$ and $[L: F]=2$. Let $\tau$ be the nontrivial automorphism of $L / F$. Choose a parabolic subgroup $P \subset G$ over $L$ of type $S_{2}$ such that $H^{\prime}=P \cap \tau(P)$ is a reductive part of $P$. It is a reductive subgroup of $G$ over $F$ whose semisimple part $\left[H^{\prime}, H^{\prime}\right]$ has type $A_{5}$ and is generated by roots $\alpha_{1}, \alpha_{3}, \ldots, \alpha_{6}$ in a proper ordering of the corresponding root system of $G$. The centralizer $H^{\prime \prime}=C_{G}\left(\left[H^{\prime}, H^{\prime}\right]\right)$ is an $F$-defined subgroup of $G$ of type $A_{1}$ containing the central torus of $H^{\prime}$. It is generated by the root subgroups $U_{ \pm \tilde{\alpha}}$ where $\tilde{\alpha}$ is the highest root.

Consider $H=H^{\prime \prime} \cdot\left[H^{\prime}, H^{\prime}\right]$. It is an $F$-defined semisimple subgroup of $G$ of type $A_{1}+A_{5}$ whose component of type $A_{1}$ is split over $L$ and is of the form $\mathbf{S L}_{1}(D)$ where $D$ is a quaternion algebra over $F$. It follows that $L \in \mathcal{A}(S B(D))$. Conversely, any splitting field of $D$ is contained in $\mathcal{A}\left(X_{S_{2}}\right)$.

Let $L_{1}$ be another quadratic extension of $F$ such that $L_{1} \in \mathcal{A}\left(X_{S_{2}}\right)$. We are going to show that $L_{1} \in \mathcal{A}(S B(D))$. Theorem 7.2 then implies that $L_{1} \sim_{S B(D)} L$ and hence $L_{1} \sim_{X_{S_{2}}} L$.

Applying the above construction to $L_{1}$ we can construct an $F$-subgroup $H_{1}$ of $G$ of type $A_{1}+A_{5}$. Its components of type $A_{1}$ correspond to quaternion algebras $D_{1}$ splitting over $L_{1}$ and it suffices to show that $D_{1}=D$.

We have that $H_{1}$ and $H$ are related to subsystems of type $A_{1}+A_{5}$ in the root system $E_{6}$. Since every two roots in the root system of type $E_{6}$ are conjugate by an element in the corresponding Weyl group $W\left(E_{6}\right)$, so are every two root subsystems of type $A_{1}+A_{5}$. Then $H_{1}, H$ are conjugate over $F_{\text {sep }}$. Let $H_{1}=g H g^{-1}$ where $g \in G\left(F_{\text {sep }}\right)$. Fixing $F$-defined maximal tori $T_{1}, T$ in $H_{1}, H$ respectively, we may assume additionally that $T_{1}=g T g^{-1}$. As $H_{1}, H, T_{1}, T$ are $F$-defined, for each $\sigma \in \operatorname{Gal}\left(F_{\text {sep }} / F\right)$ we have $a_{\sigma}=g^{-\sigma+1} \in N_{G}(H) \cap N_{G}(T)$. It follows that $H_{1}={ }^{\lambda} H$ where $\lambda=\left(a_{\sigma}\right)$; in particular we have $D_{1}={ }^{\lambda} D$.

The torus $T$ can be decomposed as $T=T^{\prime} T^{\prime \prime}$ where $T^{\prime}, T^{\prime \prime}$ are intersections of $T$ with simple components of $H$ of types $A_{1}$ and $A_{5}$ respectively. Since groups of type $A_{1}$ have no nontrivial outer automorphisms, one checks that $a_{\sigma}$ can be decomposed uniquely as $a_{\sigma}=a_{\sigma}^{\prime} a_{\sigma}^{\prime \prime}$ where $a_{\sigma}^{\prime}$ and $a_{\sigma}^{\prime \prime}$ are contained in the normalizers of $T^{\prime}$ and $T^{\prime \prime}$ in the simple components of $H$ respectively. Since the simple components of $H$ commute, $\lambda^{\prime}=\left(a_{\sigma}^{\prime}\right)$ is a cocycle with values in $\mathbf{S L}_{1}(D)$. Thus twisting of $D$ is given actually by the cocycle $\lambda^{\prime}$ and hence twisting does not change $D$ since any cocycle in $Z^{1}\left(F, \mathbf{S L}_{1}(D)\right)$ is equivalent to a cocycle with coefficients in the center $\mu_{2}$ of $\mathbf{S L}_{1}(D)$.

\subsection{Basic type $S_{3}$}

Let $L_{1}, L_{2} \in \mathcal{A}\left(X_{S_{3}}\right)$ be two quadratic extensions of $F$ contained in $\mathcal{A}\left(X_{S_{3}}\right)$. As $\mathcal{A}\left(X_{S_{3}}\right) \subset \mathcal{A}\left(X_{S_{2}}\right)$, the above construction applied to $L_{1}$ or $L_{2}$ gives us an $F$-defined subgroup $H$ of $G$ of type $A_{1}+A_{5}$. Its simple components, say $H^{\prime}$ and $H^{\prime \prime}$, are of the form $H^{\prime}=\mathbf{S L}_{1}(D)$ and $H^{\prime \prime}=\mathbf{S U}(A, \tau)$ where $D$ is a quaternion algebra over $F$ splitting over $L_{1}, L_{2}$ and $A$ is a central simple algebra of degree 6 over $K$ equipped with an involution $\tau$ of the second kind.

Any field extension $L / F$ splitting $D$ makes $H^{\prime \prime}$ a strongly inner form of a quasi-split group of type $A_{5}$, by Theorem 3.3, hence it also splits $A$. Then $[A]=\left[D \otimes_{F} K\right]$ in the Brauer group $\operatorname{Br}(K)$ and hence $H^{\prime \prime} \simeq \mathbf{S U}(T, h)$ where $T=D \otimes_{F} K$ and $h$ is a skew-hermitian form of dimension 3 given on a vector space $V$ over $T$ equipped with the standard involution, say $\sigma$, of the second kind.

We are also given that $H^{\prime \prime}$ is isotropic over $L_{1}, L_{2}$, i.e. $h$ represents zero over $L_{1}, L_{2}$. Since $L_{1}, L_{2}$ split $T$ and have degree 2 over $F$, there are 1-dimensional $T$-submodules $U_{1}, U_{2}$ in $V$ such that $h$ restricted to $U_{1}, U_{2}$ is isotropic over $L_{1}$ and $L_{2}$ respectively. This fact can be reformulated as follows. Let $v_{1} \in U_{1}$ and $v_{2} \in U_{2}$ be two nontrivial vectors. Let $\sigma_{1}, \sigma_{2}$ be involutions on $T$ corresponding to the elements $a_{1}=h\left(v_{1}, v_{1}\right)$ and $a_{2}=h\left(v_{2}, v_{2}\right)$, i.e. $\sigma_{1}, \sigma_{2}$ are compositions of $\sigma$ with the inner conjugation given by $a_{1}, a_{2}$. Then $\mathbf{S U}\left(T, \sigma_{1}\right)$ and $\mathbf{S U}\left(T, \sigma_{2}\right)$ are split over $L_{1}$ and $L_{2}$ respectively. 


\section{ZERO-CYCLES}

Clearly $\mathbf{S U}\left(T, \sigma_{1}\right)$ and $\mathbf{S U}\left(T, \sigma_{2}\right)$ are subgroups in $H^{\prime \prime}=\mathbf{S U}(T, h)$ and being groups of type $A_{1}$ they are of the form $\mathbf{S L}_{1}\left(D_{1}\right)$ and $\mathbf{S L}_{1}\left(D_{2}\right)$ where $D_{1}, D_{2}$ are quaternion algebras over $F$ contained in $T$ such that $D_{1} \otimes K=D_{2} \otimes K=T$. Recall that the pure part $D_{i}^{\circ}$ of $D_{i}$ is given by

$$
D_{i}^{\circ}=\left\{x \in T^{\circ} \mid \sigma_{i}(x)=-x\right\}=\left\{x \in D^{\circ} \otimes K \mid a_{i} \sigma(x) a_{i}^{-1}=-x\right\} .
$$

We now note that $v_{i}$ is defined up to $t_{i} \in T$, hence $D_{i}$ is defined up to conjugation in $T$. Since, by our construction, $D_{i}$ and $D$ are split over $L_{i}$, we can modify $v_{i}, a_{i}$ and $D_{i}$ (by replacing them with $t_{i} v_{i}, t_{i} a_{i} \sigma\left(t_{i}\right)$ and $t_{i} D_{i} t_{i}^{-1}$ for a proper element $\left.t_{i} \in T\right)$ such that $D \cap D_{i}$ contains a common subfield isomorphic to $L_{i}$.

As a set, this common subfield can be described as follows. As $a_{i}$ is skew with respect to $\sigma$ we can write it in the form $a_{i}=a_{i}^{\prime}+a_{i}^{\prime \prime}$ where $a_{i}^{\prime} \in K$ and $a_{i}^{\prime \prime} \in D^{\circ}$. Modifying $a_{i}$, if necessary, we may assume that $a_{i}^{\prime \prime} \neq 0$. Then we have

$$
D^{\circ} \cap D_{i}^{\circ}=\left\{d \in D^{\circ} \mid \sigma_{i}(d)=-d\right\}=\left\{d \in D^{\circ} \mid a_{i} d a_{i}^{-1}=d\right\} .
$$

It follows that $D^{\circ} \cap D_{i}^{\circ}$ has dimension 1 and is generated by $a_{i}^{\prime \prime}$.

We are ready to finish the proof. Consider a vector $v(t)=(1-t) v_{1}+t v_{2}$ where $t$ is an indeterminate. Let $a(t)=h(v(t), v(t))$. Write $a(t)=a(t)^{\prime}+a(t)^{\prime \prime}$ as above. Here $a(t)^{\prime} \in K \otimes_{F} F(t)$ and $a(t)^{\prime \prime} \in D^{\circ} \otimes_{F} F(t)$. Clearly, $a(t)^{\prime \prime}$ generates a maximal subfield $L(t)$ in $D \otimes_{F} F(t)$ splitting $D \otimes_{F} F(t)$ and making $H^{\prime \prime}$ isotropic. This implies that $L(t) \in \mathcal{A}\left(X_{S_{3}}\right)$. Since $v(0)=v_{1}$ and $v(1)=v_{2}$, arguing as in Theorem 9.2 we see that $L(t)$ has two discrete valuations with residue fields isomorphic to $L_{1}, L_{2}$.

\section{Type $E_{7}$}

By Tits' classification [Tit66], if $G$ is isotropic over an extension $L / F$ but not split, then its Tits index is one of the following:

(a)

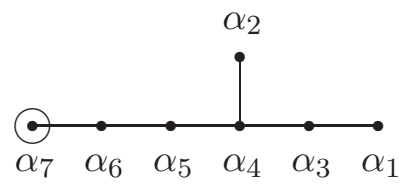

(c)

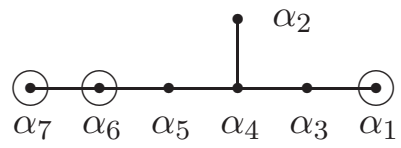

(b)

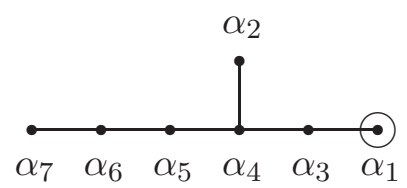

Thus we need to consider the following basic types only:

$$
S_{1}=\left\{\alpha_{1}, \ldots, \alpha_{6}\right\}, \quad S_{2}=\left\{\alpha_{2}, \ldots, \alpha_{7}\right\}, \quad S_{3}=\left\{\alpha_{2}, \ldots, \alpha_{5}\right\}, \quad S_{4}=\emptyset .
$$

If $p=3$, then we can repeat the same argument as in the case of type ${ }^{1} E_{6}$. Consider the case $p=2$.

Lemma 17.1. Let $F$ be 2-special. Then we have $n\left(S_{1}\right)=n\left(S_{2}\right)=n\left(S_{3}\right)=2$.

Proof. By [Tit92], $G$ contains an $F$-defined subgroup $H$ of type $E_{6}$. It is a strongly inner form of a quasi-split group of type $E_{6}$, since $F$ is 2 -special. We know that any such a group (and hence $G$ ) has rank at least 2 over a proper quadratic extension $E / F$. The above diagrams show that $X_{S_{i}}(E) \neq \emptyset$ for $i=1,2,3$.

By Lemma 15.3, if $E / F$ is a quadratic extension such that $X_{S_{1}}(E) \neq \emptyset$, then $X_{S_{3}}(E) \neq \emptyset$. Hence it suffices to consider types $S_{2}, S_{3}$ only. 


\section{Chernousov and A. Merkurjev}

\subsection{Basic type $S_{2}$}

Repeating verbatim the argument in $\S 16.6$ we find that $G$ contains an $F$-defined subgroup $H$ of type $A_{1}+D_{6}$ such that $\mathcal{A}\left(X_{S_{2}}\right)=\mathcal{A}(S B(D))$ where $D$ is a quaternion algebra over $F$ corresponding to the simple component of $H$ of type $A_{1}$. The connectedness of $\mathcal{A}\left(S_{2}\right)$ follows from Theorem 7.2.

\subsection{Basic type $S_{3}$}

Let $L / F$ be a quadratic extension belonging to $\mathcal{A}\left(X_{S_{3}}\right)$. Let $H_{1}$ and $H_{2}$ be the simple components of types $A_{1}$ and $D_{6}$ respectively of the group $H$ constructed in $\S 17.1$. We have $H_{1} \simeq \mathbf{S L}_{1}(D)$ where $D$ is a quaternion algebra over $F$. The above Tits diagrams show that the anisotropic $L$-kernel of $H_{2}$ is of the form $\operatorname{Spin}(f)$ where $f$ is a 4-Pfister form. It follows that up to isogeny $H$ is of the form $\mathbf{S U}(T, h)$ where $T$ is a quaternion algebra over $F$ equipped with the standard symplectic involution $\tau$ and $h$ is a skew-hermitian form with respect to $\tau$ defined over a six-dimensional vector space $V$ over $T$.

LEMma 17.2. If $T$ is nontrivial, then we have $D=T$.

Proof. If $E / F$ is an extension splitting $D$, then $H_{2}$ viewed over $E$ is a strongly inner form of a split group of type $D_{6}$. Hence $H_{2}$ is of the form $\operatorname{Spin}(g)$ where $g$ is a six-dimensional quadratic form from $I^{3}$. It follows that $T$ is split over $E$. As this is true for all splitting fields of $D$, the quaternion algebras $D$ and $T$ coincide.

Let $Y$ be the involution variety corresponding to $\mathrm{SO}(D, h)$. By our construction, $L \in \mathcal{A}(Y)$. Conversely, let $E \in \mathcal{A}(Y)$. We noted above that $H_{2}$ viewed over $E$ is related to the quadratic form $g$ of dimension 12 from $I^{3}$. Since $g$ is isotropic over $E$ and since 10-dimensional quadratic forms from $I^{3}$ are isotropic, we conclude that the $E$-rank of $H_{2}$ is at least 2. This implies that the $E$-rank of $G$ is at least 2. An inspection of the above Tits diagrams shows that $E \in \mathcal{A}\left(X_{S_{3}}\right)$. Thus we showed that $\mathcal{A}(Y)=\mathcal{A}\left(X_{S_{3}}\right)$ and the connectedness of $\mathcal{A}(Y)$ follows from Theorem 10.3.

\section{Two examples of a nontrivial $\overline{\mathrm{CH}}_{0}(X)$}

In this section we give two examples of projective homogeneous varieties $X$ with nontrivial group $\overline{\mathrm{CH}}_{0}(X)$.

\subsection{First example}

We owe to Vishik the observation that [KM90] essentially contains an example. Let $F$ be a field of characteristic different from 2 and let $a, b \in F^{\times}$. Let $Q_{a, b}$ be the quadric of the 2-fold Pfister form $\langle\langle a, b\rangle\rangle=\langle 1,-a,-b, a b\rangle$. In the split case (over a quadratic extension $E / F$ ), $Q_{a, b}$ is isomorphic to $\mathbb{P}_{F}^{1} \times \mathbb{P}_{F}^{1}$; in particular, there are two families of lines on $Q_{a, b}$. Let $l_{a, b}$ be one of these lines and denote by $u_{a, b} \in \mathrm{CH}_{1}\left(Q_{a, b}\right)$ the push-forward of the class of $l_{a, b}$ under the morphism $\left(Q_{a, b}\right)_{E} \rightarrow Q_{a, b}$.

Let $X_{a, b}$ be the variety of isotropic planes of $\langle\langle a, b\rangle\rangle$. Over the field $E \in \mathcal{A}\left(X_{a, b}\right)$, the variety $X_{a, b}$ is the disjoint union of two copies of the projective line. We can view $l_{a, b}$ as a rational point of $X_{a, b}$ over $E$. Denote the push-forward of the class of $l_{a, b}$ in $\mathrm{CH}_{0}\left(X_{a, b}\right)$ by $x_{a, b}$, so $x_{a, b}$ is a zerocycle of degree 2 . The incidence correspondence between $Q_{a, b}$ and $X_{a, b}$ induces a map $\mathrm{CH}_{0}\left(X_{a, b}\right) \rightarrow$ $\mathrm{CH}_{1}\left(Q_{a, b}\right)$ taking $u_{a, b}$ to $x_{a, b}$.

Let $q=\langle 1,-a,-b, a b,-c,-d, c d\rangle$ for some $a, b, c, d \in F^{\times}$and let $Q$ be the corresponding fivedimensional quadric. We have natural embeddings $Q_{a, b} \hookrightarrow Q$ and $Q_{c, d} \hookrightarrow Q$. Denote the images of $u_{a, b}$ and $u_{c, d}$ in $\mathrm{CH}_{1}(Q)$ by the same symbols.

It is shown in [KM90, Corollary 5.2] that, in the case $-1 \in F^{\times 2}$, the classes $u_{a, b}$ and $u_{c, d}$ are equal if and only if the Pfister form $\langle\langle a, b, c, d\rangle\rangle$ is isotropic. 


\section{ZERO-CYCLES}

Denote by $X$ the variety of isotropic planes of $q$. The images of $x_{a, b}$ and $x_{c, d}$ in $\mathrm{CH}_{0}(X)$ will be denoted by the same symbols. We have the incidence correspondence between $Q$ and $X$ that induces a homomorphism $\mathrm{CH}_{0}(X) \rightarrow \mathrm{CH}_{1}(Q)$ taking $x_{a, b}-x_{c, d}$ to $u_{a, b}-u_{c, d}$.

If $F$ is a field such that $-1 \in F^{\times 2}$ and the Pfister form $\langle\langle a, b, c, d\rangle\rangle$ is anisotropic, the class $u_{a, b}-u_{c, d}$ is nontrivial and therefore $x_{a, b}-x_{c, d} \neq 0$. As $\operatorname{deg}\left(x_{a, b}-x_{c, d}\right)=0$, the class $x_{a, b}-x_{c, d}$ represents a nontrivial element of $\overline{\mathrm{CH}}_{0}(X)$. Note that $X$ corresponds to the subset $\left\{\alpha_{1}, \alpha_{3}\right\}$ of the set of vertices of the Dynkin diagram $B_{3}$.

\subsection{Second example}

We shall give an example of a three-dimensional projective homogeneous variety $X$ with a nontrivial group $\overline{\mathrm{CH}}_{0}(X)$. Note that this is an example of the smallest possible dimension in view of Proposition 4.4.

Let $C_{i}, i=1,2,3$, be three conic curves corresponding to quaternion algebras $Q_{i}$ over $F$ and let $X=C_{1} \times C_{2} \times C_{3}$. For every subset $S \subset\{1,2,3\}$ let $Q_{S}$ be the tensor product of $Q_{i}$ for all $i \in S$ (in particular, $Q_{\emptyset}=F$ ). We define the complex

$$
\coprod_{S \neq \emptyset} K_{1}\left(Q_{S}\right) \stackrel{\alpha}{\rightarrow}\left(F^{\times}\right)^{3} \stackrel{\beta}{\rightarrow} H^{3}(F, \mathbb{Z} / 2 \mathbb{Z})
$$

by the following formulas:

$$
\begin{gathered}
\alpha(x)=\left(a_{1}, a_{2}, a_{3}\right), \quad a_{i}=\prod_{S \ni i} \operatorname{Nrd}_{S}\left(x_{S}\right), \\
\beta\left(a_{1}, a_{2}, a_{3}\right)=\left(a_{1}\right) \cup\left[Q_{1}\right]+\left(a_{2}\right) \cup\left[Q_{2}\right]+\left(a_{3}\right) \cup\left[Q_{3}\right],
\end{gathered}
$$

where $\operatorname{Nrd}_{S}: K_{1}\left(Q_{S}\right) \rightarrow K_{1}(F)=F^{\times}$is the reduced norm homomorphism, (a) denotes the class in $H^{1}(F, \mathbb{Z} / 2 \mathbb{Z})$ corresponding to $a \in F^{\times}$under the Kummer isomorphism, and $[Q]$ denotes the class of $Q$ in $H^{2}(F, \mathbb{Z} / 2 \mathbb{Z})$.

Proposition 18.1. The group $\overline{\mathrm{CH}}_{0}(X)$ is isomorphic to the homology group of the complex (18.1).

Proof. In the spectral sequence (4.1) for $X$ the only possibly nontrivial differential coming to $E_{*}^{3,-3}$ is $d_{2}: A^{1}\left(X, K_{2}\right)=E_{2}^{1,-2} \rightarrow E_{2}^{3,-3}=\mathrm{CH}_{0}(X)$ and all the differentials coming to $E_{*}^{1,-2}$ are trivial. Therefore, the sequence

$$
K_{1}(X)^{(1)} \rightarrow A^{1}\left(X, K_{2}\right) \stackrel{d_{2}}{\longrightarrow} \mathrm{CH}_{0}(X) \stackrel{e^{\prime}}{\rightarrow} K_{0}(X)
$$

is exact. As the image of $d_{2}$ coincides with $\operatorname{Ker}\left(e^{\prime}\right)=\overline{\mathrm{CH}}_{0}(X)$, it is sufficient to identify the cokernel of the first homomorphism in the sequence with the homology group of the complex (18.1).

The group $A^{1}\left(X, K_{2}\right)$ was computed in [Mer95a]. If all $Q_{i}$ are split, we have

$$
A^{1}\left(X, K_{2}\right)=K_{1}(F) \otimes \mathrm{CH}^{1}(X) \simeq F^{\times} \otimes \mathbb{Z}^{3}=\left(F^{\times}\right)^{3},
$$

where we identify $\mathrm{CH}^{1}(X)$ with $\operatorname{Pic}(X)=\mathbb{Z} h_{1} \oplus \mathbb{Z} h_{2} \oplus \mathbb{Z} h_{3} \simeq \mathbb{Z}^{3}$ (here $h_{i}$ is the class of a rational point in $\left.C_{i}\right)$. In the general case the group $A^{1}\left(X, K_{2}\right)$ is canonically isomorphic to a subgroup of $\left(F^{\times}\right)^{3}$, namely to the kernel of the homomorphism $\beta$.

The group $K_{1}(X)$ was computed in [Qui73, $\S 8$, Theorem 4.1]:

$$
K_{1}(X)=\coprod_{S} K_{1}\left(Q_{S}\right)
$$

The first term of the topological filtration $K_{1}(X)^{(1)}$ is the kernel of the natural homomorphism $K_{1}(X) \rightarrow K_{1} F(X)$. We claim that, for every $S$, the corresponding homomorphism $K_{1}\left(Q_{S}\right) \rightarrow$ $K_{1} F(X)$ is the reduced norm map $\operatorname{Nrd}_{S}$ followed by the natural homomorphism $K_{1} F \rightarrow K_{1} F(X)$. 


\section{Chernousov and A. Merkurjev}

Since the group $K_{1}$ of a field injects when the field gets extended, we may assume that all $Q_{i}$ are split. In this case the reduced norm $\mathrm{Nrd}_{S}$ is an isomorphism and (18.2) identifies $K_{1}(X)$ with the direct sum of $K_{1}(F) L_{S}$, where $L_{S}$ is the tensor product of the canonical line bundles $L_{i}$ on the $C_{i} \simeq \mathbb{P}_{F}^{1}$ such that $i \in S$. As $L_{S}$ maps to 1 in $K_{0} F(X)$, the claim follows.

We have shown that $K_{1}(X)^{(1)}$ consists of all $x=\left(x_{S}\right)$ such that $\sum \operatorname{Nrd}_{S}\left(x_{S}\right)=0$. We claim that the image of $x$ in $A^{1}\left(X, K_{2}\right) \subset\left(F^{\times}\right)^{3}$ is equal to $\left(a_{1}, a_{2}, a_{3}\right)$, where $a_{i}$ is the product of $\operatorname{Nrd}_{S}\left(x_{S}\right)$ for all $S \ni i$. We can assume that all $Q_{i}$ are split. Under the identification of $K_{1}\left(Q_{S}\right)$ with $K_{1}(F)$, the element $x$ corresponds to $\sum b_{S} L_{S}=\sum_{S \neq \emptyset} b_{S}\left(L_{S}-1\right)$, where $b_{S}=\operatorname{Nrd}_{S}\left(x_{S}\right)$. Note that the image of $L_{S}-1$ under the natural map $K_{0}(X)^{(1)} \rightarrow \mathrm{CH}^{1}(X)$ is equal to the first Chern class $c_{1}\left(L_{S}\right)=\sum_{i \in S} c_{1}\left(L_{i}\right)=\sum_{i \in S} h_{i}$. Therefore, the image of $x$ in $A^{1}\left(X, K_{2}\right)=\mathbb{Z} h_{1} \oplus \mathbb{Z} h_{2} \oplus \mathbb{Z} h_{3}$ is equal to $a_{1} h_{1} \oplus a_{2} h_{2} \oplus a_{3} h_{3}$, where $a_{i}$ is the product of $b_{S}$ for all $S \ni i$.

It follows from the claim that the image of the homomorphism $K_{1}(X)^{(1)} \rightarrow A^{1}\left(X, K_{2}\right) \subset\left(F^{\times}\right)^{3}$ is equal to $\operatorname{Im} \alpha$.

Example 18.2. Let $F=k((t))$ be the Laurent power series field over a field $k$ of characteristic different from $2, Q_{i}=\left(b_{i},-t\right)_{F}$ for some $b_{i} \in k^{\times}$. Let $b_{S}$ be the product of $b_{i}$ for all $i \in S, k_{S}=k\left(b_{S}^{1 / 2}\right)$ and $\operatorname{Norm}_{S}: k_{S}^{\times} \rightarrow k^{\times}$be the norm homomorphism. Since $\left(t^{n}, t^{m}, t^{p}\right) \in \operatorname{Im}(\alpha)$ for all $n, m, p$, $H^{p}(F, \mathbb{Z} / 2 \mathbb{Z})$ is equal to $H^{p}(k, \mathbb{Z} / 2 \mathbb{Z}) \oplus H^{p-1}(k, \mathbb{Z} / 2 \mathbb{Z}) \cup(-t)$ and $\operatorname{Im}_{\operatorname{Nrd}} \operatorname{Im}_{S}=\operatorname{Iorm}_{S} \cdot F^{\times 2} \cup$ $\operatorname{Im} \operatorname{Norm}_{S} \cdot t \cdot F^{\times 2}$, the homology group of (18.1) is isomorphic to the homology group of the complex

$$
\coprod_{S \neq \emptyset} k_{S}^{\times} \stackrel{\alpha^{\prime}}{\longrightarrow}\left(k^{\times}\right)^{3} \stackrel{\beta^{\prime}}{\longrightarrow} H^{2}(k, \mathbb{Z} / 2 \mathbb{Z}),
$$

where

$$
\begin{gathered}
\alpha^{\prime}(x)=\left(a_{1}, a_{2}, a_{3}\right), \quad a_{i}=\prod_{S \ni i} \operatorname{Norm}_{S}\left(x_{S}\right), \\
\beta^{\prime}\left(a_{1}, a_{2}, a_{3}\right)=\left(a_{1}\right) \cup\left(b_{1}\right)+\left(a_{2}\right) \cup\left(b_{2}\right)+\left(a_{3}\right) \cup\left(b_{3}\right) .
\end{gathered}
$$

There is a field $k$ and the elements $b_{i}$ so that the complex is not acyclic (see [STW82, $\left.\S 5\right]$ ) and therefore $\overline{\mathrm{CH}}_{0}(X) \neq 0$ in this case.

\section{REFERENCES}

Che94 V. Chernousov, Remark on (mod 5)-Serre's invariant for groups of type $E_{8}$, Mat. Zametki 56 (1994), 116-121.

Che03 V. Chernousov, The kernel of the Rost invariant, Serre's conjecture II and the Hasse principle for quasi-split groups ${ }^{3,6} D_{4}, E_{6}, E_{7}$, Math. Ann. 326 (2003), 297-330.

CT99 V. Chernousov and L. Timoshenko, On the group of R-equivalence classes of semisimple groups over arithmetic fields, Algebra i Analiz 11 (1999), no. 6, 191-221.

CGM05 V. Chernousov, S. Gille and A. Merkurjev, Motivic decomposition of isotropic projective homogeneous varieties, Duke Math. J. 126 (2005), 137-159.

Gar01 S. Garibaldi, The Rost invariant has trivial kernel for quasi-split groups of low rank, Comment. Math. Helv. 76 (2001), 684-711.

GMS03 S. Garibaldi, A. Merkurjev and J.-P. Serre, Cohomological invariants in Galois cohomology, University Lecture Series, vol. 28 (American Mathematical Society, Providence, RI, 2003).

Her94 I. N. Herstein, Noncommutative rings, Reprint of the 1968 original, with an afterword by Lance W. Small (Mathematical Association of America, Washington, DC, 1994). 


\section{ZERO-CYCLES}

Jac68 N. Jacobson, Structure and representations of Jordan algebras, American Mathematical Society Colloquium Publications, vol. 39 (American Mathematical Society, Providence, RI, 1968).

Kar91 N. Karpenko, Algebro-geometric invariants of quadratic forms, Leningrad (St. Petersburg) Math. J. 2 (1991), 119-138.

KM90 N. A. Karpenko and A. S. Merkurjev, Chow groups of projective quadrics, Algebra i Analiz 2 (1990), no. 3, 218-235.

KMRT98 M.-A. Knus, A. Merkurjev, M. Rost and J.-P. Tignol, The book of involutions, with a preface in French by J. Tits (American Mathematical Society, Providence, RI, 1998).

Kra05 D. Krashen, Zero cycles on homogeneous varieties, Preprint (2006), math.AG/0501399.

Mer95a A. S. Merkurjev, The group $H^{1}\left(X, \mathcal{K}_{2}\right)$ for projective homogeneous varieties, Algebra i Analiz 7 (1995), no. 3, 136-164.

Mer95b A. S. Merkurjev, Zero-dimensional cycles on some involutive varieties, Zap. Nauchn. Sem. S.-Peterburg. Otdel. Mat. Inst. Steklov. (POMI) 227 (1995), Voprosy Teor. Predstav. Algebr i Grupp. 4, 93-105, 158.

MS82 A. S. Merkurjev and A. A. Suslin, K-cohomology of Severi-Brauer varieties and the norm residue homomorphism, Izv. Akad. Nauk SSSR Ser. Mat. 46 (1982), 1011-1046, 1135-1136.

Pan84 I. Panin, Application of K-theory in algebraic geometry, Thesis, LOMI, Leningrad (1984).

Pan94 I. A. Panin, On the algebraic K-theory of twisted flag varieties, K-Theory 8 (1994), 541-585.

PR84 H. P. Petersson and M. L. Racine, Cubic subfields of exceptional simple Jordan algebras, Proc. Amer. Math. Soc. 91 (1984), 31-36.

PR95 H. Petersson and M. Racine, On the invariants mod 2 of Albert algebras, J. Algebra 174 (1995), 1049-1072.

PSZ05 V. Petrov, N. Semenov and K. Zainoulline, Zero-cycles on twisted Cayley plane, Preprint (2005), http://www.math.uni-bielefeld.de/lag/.

PR94 V. Platonov and A. Rapinchuk, Algebraic groups and number theory (Academic Press, New York, 1994).

Qui73 D. Quillen, Higher algebraic K-theory, I, Lecture Notes in Mathematics, vol. 341 (Springer, Berlin, 1973), 85-147.

Rac72 M. Racine, A note on quadratic Jordan algebras of degree 3, Trans. Amer. Math. Soc. 164 (1972), 93-103.

Ros96 M. Rost, Chow groups with coefficients, Doc. Math. 1 (1996), no. 16, 319-393 (electronic).

STW82 D. B. Shapiro, J.-P. Tignol and A. R. Wadsworth, Witt rings and Brauer groups under multiquadratic extensions, II, J. Algebra 78 (1982), 58-90.

Spr60 T. A. Springer, The classification of reduced exceptional simple Jordan algebras, Indag. Math. 22 (1960), 414-422.

Ste65 R. Steinberg, Regular elements of semisimple groups, Publ. Math. Inst. Hautes Études Sci. 25 (1965), 281-312.

Swa89 R. G. Swan, Zero cycles on quadratic hypersurfaces, Proc. Amer. Math. Soc. 107 (1989), 43-46

Tit66 J. Tits, Classification of algebraic semisimple groups, in Algebraic groups and discontinuous subgroups (eds A. Borel and G. D. Mostow), Proceedings of Symposia in Pure Mathematics, vol. 9 (American Mathematical Society, Providence, RI, 1966), 33-62.

Tit68 J. Tits, Formes quadratiques, groupes orthogonaux et algèbres de Clifford, Invent. Math. 5 (1968), $19-41$.

Tit90 J. Tits, Strongly inner anisotropic forms of simple algebraic groups, J. Algebra 131 (1990), 648-677.

Tit92 J. Tits, Sur les degrés des extensions de corps déployant les groupes algébriques simples, C. R. Acad. Sci. 315 (1992), 1131-1138. 


\section{ZERO-CYCLES}

Vladimir Chernousov chernous@math.ualberta.ca

Department of Mathematical Sciences, University of Alberta, Edmonton, Alberta, Canada T6G 2G1

Alexander Merkurjev merkurev@math.ucla.edu

Department of Mathematics, University of California, Los Angeles, CA 90095-1555, USA 